YITP-SB 13-21

\title{
Complete spectrum and scalar products for the open spin-1/2 XXZ quantum chains with non-diagonal boundary terms
}

\author{
S. Faldella 1 , N. Kitanine², G. Niccoli ${ }^{3}$
}

\begin{abstract}
We use the quantum separation of variable (SOV) method to construct the eigenstates of the open XXZ chain with the most general boundary terms. The eigenstates in the inhomogeneous case are constructed in terms of solutions of a system of quadratic equations. This SOV representation permits us to compute scalar products and can be used to calculate form factors and correlation functions.
\end{abstract}

\footnotetext{
${ }^{1}$ IMB, UMR 5584 du CNRS, Université de Bourgogne, France, Simone.Faldella@u-bourgogne.fr ${ }^{2}$ IMB, UMR 5584 du CNRS, Université de Bourgogne, France, Nikolai.Kitanine@u-bourgogne.fr

${ }^{3}$ YITP, Stony Brook University, New York 11794-3840, USA, niccoli@max2.physics.sunysb.edu; etc
} 


\section{Introduction}

Quantum integrable models and, in particular the spin chains, provide an extremely important tool for the non-perturbative analysis of quantum systems. The study of these models starting with the introduction of the Bethe ansatz [5] led to a number of important predictions for the equilibrium one-dimensional quantum systems. Recently it was shown that the quantum spin chains can be also used to describe several nontrivial physical phenomena in the out-of-equilibrium case. However the applications of the Bethe ansatz techniques to the systems out of equilibrium turned out to be much more complicated.

One of the simplest examples of a quantum integrable model out of equilibrium is the XXZ spin chain with non-diagonal boundary terms. The study of this system is necessary to tackle a wide range of open problems from the relaxation behavior of some classical stochastic processes, such as the ASEP [15, 16] (asymmetric simple exclusion processes), to the transport properties of the quantum spin systems [43, 42. In particular, it should lead to a better understanding of the diffusive spin transport in the spin chains.

It turns out that most of these problems require computation of correlation functions for the corresponding models. For the equilibrium case an efficient method of computation of the correlation functions based on the quantum inverse scattering method (QISM) and the algebraic Bethe ansatz [21] was first established for the periodic spin chains [30, 31] and then for the open spin chains with parallel boundary magnetic fields [26, 27]. Recent advances of this technique have been essential in order to study the asymptotic behavior of the two-point dynamical correlation functions and structure factors [28, 29]. The most crucial steps of this approach are the quantum inverse problem solution [30, 32] and the computation of the scalar products of the so-called on-shell and off-shell Bethe vectors [47. For the quantum systems solvable by the algebraic Bethe ansatz these two steps lead to manageable expressions for the correlation functions.

It is important to mention that the usual algebraic Bethe ansatz technique for open systems 44] (based on the Cherednik reflection equation [12]) cannot be applied directly to the spin chains with non-diagonal boundary terms (which can be understood as non-parallel boundary magnetic fields). The first successful attempt to describe the spectrum of the XXZ spin chain with nondiagonal boundary terms was performed by Nepomechie [34, 35] using the Baxter T-Q equation. This method worked only for the roots of unity points and only if the boundary terms satisfied a very particular constraint relating the magnetic fields on the left and right boundaries. Similar constraint was obtained in [7] within the framework of the generalized algebraic Bethe ansatz. Using a very particular gauge transformation (inspired by the Baxter [3, 4] and Faddeev-Takhtadjan [49] approaches for the XYZ spin chain) the authors constructed for the first time the eigenstates of the XXZ spin chain with non-parallel boundary magnetic fields. It is important to mention that this approach provided a possibility to get rid of the requirement to consider only the spin chains at the roots of unity. A slightly different version of this technique, based on the vertex-IRF transformation, was proposed in [50]. Even though this method offered a more clear algebraic construction, it required one more additional constraint for the boundary parameters.

A priori this last method appeared to be the most suitable for the study of correlation functions, as some crucial quantities could be computed explicitly within this framework [23]. However it turns out that the computation of the scalar products of Bethe vectors remains an open problem despite

\footnotetext{
${ }^{1}$ In this case with boundary constrain alternative methods leading to Bethe equation formulations have been developed both in [18, 41, in the Temperley-Lieb algebraic framework, and in [13, 14], by combing coordinate Bethe ansatz and matrix ansatz.
} 
several unsuccessful attempts to solve it. Moreover, it seems that the boundary constraints remain essential to apply the algebraic Bethe ansatz in the non-diagonal.

Other approaches have been developed to deal with this spectral problem in this general setting. For the eigenvalue characterization in [17] a new functional method has been introduced leading to nested Bethe ansatz type equations similar to those presented previously in [33] in the generalized $T$ $Q$ formalism. In the so-called $q$-Onsager formalism the eigenstate construction has been addressed in [2, 1] leading to a characterization of the spectrum in terms of the roots of some characteristic polynomials.

An alternative way to construct eigenvalues and eigenvectors for quantum integrable systems not solvable by the algebraic Bethe ansatz is the quantum separation of variables (SOV) introduced by Sklyanin [45, 46] for the quantum Toda chain. It was recently shown that besides the spectrum the scalar products of Bethe vectors can also be computed using the SOV technique leading to manageable expressions for the matrix elements of local operators for the cyclic sine-Gordon model, anti-periodic spin chains, SOS model and several other systems [40, 19, 20, 37, 38, 39].

Recently this method was also applied to the open spin chains with non-diagonal boundary terms [36]. This technique provided a possibility to construct the spectrum and to compute scalar products for a spin chain with non-diagonal boundaries under condition that one of boundary $K$ matrices is triangular. While this conditions also represents a constraint its nature is quite different from ones used for other approaches. In particular, it is a constraint only for one boundary (and not relating parameters for two boundaries). It is worth mentioning that for the XXX chain even the most general case can be reduced to this one due to the $S U(2)$ invariance of the bulk Hamiltonian. Therefore the SOV approach can be used to completely solve the XXX chain with the most general non-diagonal boundary fields. In fact, some results in this direction for this model already appear in [24, 25], where the functional version of the separation of variables of Sklyanin has been developed under general boundary conditions leading to the eigenvalues and wave-function characterizations. This functional approach, however, does not lead to the construction of the transfer matrix eigenstates in the original Hilbert space of the quantum chain, which instead can be obtained adapting to the rational 6 -vertex case the SOV method developed in [36. However, the $S U(2)$ symmetry is lost for the XXZ case and the remaining $U(1)$ symmetry is not sufficient (in general) to triangularize one of the $K$ matrices.

In this paper we study the quantum XXZ spin chains with the most general boundary terms. To construct the eigenstates and to compute the spectrum of the teansfer matrix we use two techniques: first we apply the gauge transformation introduced in [7] and then we apply the SOV approach. The gauge transformation provides a possibility to obtain a triangular boundary $K$ matrix which leads to a SOV solution similar to that of [36. These two steps combined lead to the description of the spectrum and the eigenstates for the most general open XXZ chain and to determinant representations for the scalar product of such SOV states.

It is important to underline that this solution works if the boundary constraint is not satisfied. More precisely, there are two equivalent ways to construct the eigenstates. If the constraint is satisfied one of them does not work.

The main peculiarity of this case is the fact that the SOV analysis does not lead to a polynomial $Q$ operator and hence to the Bethe equations. Here the eigenstates are defined through a system of quadratic equations. These equations replace the Baxter $T-Q$ relation and permit us to completely characterize the eigenstates and the eigenvalues of the transfer matrix.

The main advantage of this approach is the simplicity of the final representation for the eigenstates in the SOV basis. On the other hand the main difficulty is the fact that this approach 
works only for the inhomogeneous case and the thermodynamic and homogeneous limits cannot be taken easily. In particular, it is not yet clear how to identify the ground state for the hermitian Hamiltonian and the stationary state for the model out of equilibrium. However we think that these problems can be solved within the framework of our approach.

The paper is organized as follows. In the Section 2 we describe the reflection algebra and construction of the commuting transfer matrices following [44]. In the Section 3 we introduce the gauge transformation and establish the main properties of the gauged elements of the monodromy matrix. The SOV basis in terms of these operators is constructed in the Section 4. The main result of the paper is given in the Section 5 where we construct the eigenstates of the transfer matrix in the SOV framework. In the last section we give our expression for scalar products. The implications of these results and some open problems are discussed in the conclusion. In the appendix we give the explicit form of the gauged transformed boundary matrices.

\section{Reflection algebra and open spin-1/2 XXZ quantum chain}

We study in this paper the quantum open XXZ spin chain with the most general boundary terms:

$$
\begin{aligned}
H & =\sum_{i=1}^{\mathrm{N}-1}\left(\sigma_{i}^{x} \sigma_{i+1}^{x}+\sigma_{i}^{y} \sigma_{i+1}^{y}+\cosh \eta \sigma_{i}^{z} \sigma_{i+1}^{z}\right) \\
& +\frac{\sinh \eta}{\sinh \zeta_{-}}\left[\sigma_{1}^{z} \cosh \zeta_{-}+2 \kappa_{-}\left(\sigma_{1}^{x} \cosh \tau_{-}+i \sigma_{1}^{y} \sinh \tau_{-}\right)\right] \\
& +\frac{\sinh \eta}{\sinh \zeta_{+}}\left[\left(\sigma_{\mathrm{N}}^{z} \cosh \zeta_{+}+2 \kappa_{+}\left(\sigma_{\mathrm{N}}^{x} \cosh \tau_{+}+i \sigma_{\mathrm{N}}^{y} \sinh \tau_{+}\right)\right.\right.
\end{aligned}
$$

This Hamiltonian acts in a tensor product $\mathbb{C}^{2 \otimes N}, \sigma_{i}^{a}$ are local spin $1 / 2$ operators (Pauli matrices), $\Delta=\cosh \eta$ is the anisotropy parameter and six complex boundary parameters $\zeta_{ \pm}, \kappa_{ \pm}$and $\tau_{ \pm}$give the most general boundary interactions.

In the framework of the quantum inverse scattering method the open XXZ spin chain is characterized by monodromy matrices $\mathcal{U}(\lambda)$ which are solutions of the following reflection equation:

$$
R_{12}(\lambda-\mu) \mathcal{U}_{1}(\lambda) R_{21}(\lambda+\mu-\eta) \mathcal{U}_{2}(\mu)=\mathcal{U}_{2}(\mu) R_{12}(\lambda+\mu-\eta) \mathcal{U}_{1}(\lambda) R_{21}(\lambda-\mu),
$$

where the $R$-matrix is the 6-vertex trigonometric solution of the Yang-Baxter equation:

$$
R_{12}(\lambda-\mu) R_{13}(\lambda) R_{23}(\mu)=R_{23}(\mu) R_{13}(\lambda) R_{12}(\lambda-\mu)
$$

and

$$
R_{12}(\lambda)=\left(\begin{array}{cccc}
\sinh (\lambda+\eta) & 0 & 0 & 0 \\
0 & \sinh \lambda & \sinh \eta & 0 \\
0 & \sinh \eta & \sinh \lambda & 0 \\
0 & 0 & 0 & \sinh (\lambda+\eta)
\end{array}\right) \in \operatorname{End}\left(\mathcal{H}_{1} \otimes \mathcal{H}_{2}\right)
$$

where $\mathcal{H}_{a} \simeq \mathbb{C}^{2}$ is a 2 -dimensional linear space. The most general scalar solution $K(\lambda) \in \operatorname{End}\left(\mathcal{H}_{0} \simeq\right.$ $\mathbb{C}^{2}$ ) of the reflection equation is the following $2 \times 2$ matrix:

$$
K(\lambda ; \zeta, \kappa, \tau)=\frac{1}{\sinh \zeta}\left(\begin{array}{cc}
\sinh (\lambda-\eta / 2+\zeta) & \kappa e^{\tau} \sinh (2 \lambda-\eta) \\
\kappa e^{-\tau} \sinh (2 \lambda-\eta) & \sinh (\zeta-\lambda+\eta / 2)
\end{array}\right)
$$


where $\zeta, \kappa$ and $\tau$ are arbitrary complex parameters.

Starting from the scalar $K$-matrix following Sklyanin [44] we can construct new solutions in the $2^{\mathrm{N}}$-dimensional representation space:

$$
\mathcal{H}=\otimes_{n=1}^{\mathrm{N}} \mathcal{H}_{n} .
$$

More precisely it is possible to construct two classes of solutions to the same reflection equation (2.2). First we define:

$$
K_{-}(\lambda)=K\left(\lambda ; \zeta_{-}, \kappa_{-}, \tau_{-}\right), \quad K_{+}(\lambda)=K\left(\lambda+\eta ; \zeta_{+}, \kappa_{+}, \tau_{+}\right),
$$

where $\zeta_{ \pm}, \kappa_{ \pm}, \tau_{ \pm}$are arbitrary complex parameters. Then we construct the (bulk) inhomogeneous "left to right" monodromy matrix

$$
M_{0}(\lambda)=R_{0 \mathrm{~N}}\left(\lambda-\xi_{\mathrm{N}}-\eta / 2\right) \ldots R_{01}\left(\lambda-\xi_{1}-\eta / 2\right)=\left(\begin{array}{cc}
A(\lambda) & B(\lambda) \\
C(\lambda) & D(\lambda)
\end{array}\right) .
$$

In a similar way we can construct the "right to left" monodromy matrix

$$
\hat{M}(\lambda)=(-1)^{\mathrm{N}} \sigma_{0}^{y} M^{t_{0}}(-\lambda) \sigma_{0}^{y} .
$$

The inhomogeneity parameters $\xi_{j}$ are arbitrary complex numbers, in this paper we need to keep them generic, the physical case corresponds to the homogeneous $\operatorname{limit} 2$. $M_{0}(\lambda) \in \operatorname{End}\left(\mathcal{H}_{0} \otimes \mathcal{H}\right)$, satisfies the Yang-Baxter relation

$$
R_{12}(\lambda-\mu) M_{1}(\lambda) M_{2}(\mu)=M_{2}(\mu) M_{1}(\lambda) R_{12}(\lambda-\mu) .
$$

Then we can define the boundary monodromy matrices $\mathcal{U}_{ \pm}(\lambda) \in \operatorname{End}\left(\mathcal{H}_{0} \otimes \mathcal{H}\right)$ as follows:

$$
\begin{aligned}
& \mathcal{U}_{-}(\lambda)=M_{0}(\lambda) K_{-}(\lambda) \hat{M}_{0}(\lambda)=\left(\begin{array}{cc}
\mathcal{A}_{-}(\lambda) & \mathcal{B}_{-}(\lambda) \\
\mathcal{C}_{-}(\lambda) & \mathcal{D}_{-}(\lambda)
\end{array}\right), \\
& \mathcal{U}_{+}^{t_{0}}(\lambda)=M_{0}^{t_{0}}(\lambda) K_{+}^{t_{0}}(\lambda) \hat{M}_{0}^{t_{0}}(\lambda)=\left(\begin{array}{ll}
\mathcal{A}_{+}(\lambda) & \mathcal{C}_{+}(\lambda) \\
\mathcal{B}_{+}(\lambda) & \mathcal{D}_{+}(\lambda)
\end{array}\right) .
\end{aligned}
$$

$\mathcal{U}_{-}(\lambda)$ and $\mathcal{V}_{+}(\lambda)=\mathcal{U}_{+}^{t_{0}}(-\lambda)$ are two classes of solutions of the reflection equation (2.2). It is shown by Sklyanin [44 that from this couple of monodromy matrices one can define the following commuting family of transfer matrices:

$$
\mathcal{T}(\lambda)=\operatorname{tr}_{0}\left\{K_{+}(\lambda) M(\lambda) K_{-}(\lambda) \hat{M}(\lambda)\right\}=\operatorname{tr}_{0}\left\{K_{+}(\lambda) \mathcal{U}_{-}(\lambda)\right\}=\operatorname{tr}_{0}\left\{K_{-}(\lambda) \mathcal{U}_{+}(\lambda)\right\} \in \operatorname{End}(\mathcal{H}) .
$$

In this paper we characterize the complete spectrum (eigenvalue \& eigenstates) of this transfer matrix for the most general class of non-diagonal boundary $K$-matrices in this way generalizing the results of [36]. Our analysis applies also to the open spin-1/2 XXZ quantum chain with the most general non-diagonal boundary terms (2.1), as this Hamiltonian is obtained in the homogeneous limit by the following derivative of the transfer matrix (2.13):

$$
H=\frac{2(\sinh \eta)^{1-2 N}}{\operatorname{tr}\left\{K_{+}(\eta / 2)\right\} \operatorname{tr}\left\{K_{-}(\eta / 2)\right\}} \frac{d}{d \lambda} \mathcal{T}(\lambda)_{\lambda=\eta / 2}+\text { constant. }
$$

\footnotetext{
${ }^{2}$ Here the homogeneous limit corresponds to $\xi_{m}=0$ for $m=1, \ldots, \mathrm{N}$.
} 
If the boundary $K$ matrices are diagonal $\left(\kappa_{ \pm}=0\right)$ the eigenstates of the transfer matrix can be constructed using the algebraic Bethe ansatz [44]. In the non-diagonal case it turns out to be impossible as the ferromagnetic state (for example with all the spins up) is no more the highest weight vector for the reflection algebra. Several methods were applied to overcome this difficulty. In particular, in [7] the authors proposed a gauge transformation to diagonalize one of the $K$ matrices and to make the second one triangular. Such gauge transformation exists only if the boundary parameters satisfy a boundary constraint [34]. Our goal here is to construct the eigenstates for the most general values of the boundary parameters.

\subsection{First fundamental properties}

Here we establish some properties of the generators $\mathcal{A}_{-}(\lambda), \mathcal{B}_{-}(\lambda), \mathcal{C}_{-}(\lambda)$ and $\mathcal{D}_{-}(\lambda)$ of the reflection algebra which play a fundamental role in the solution of the transfer matrix $\mathcal{T}(\lambda)$ spectral problem.

First of all their commutation relations follow from the reflection equation (2.2). Using these relations it was shown by Sklyanin that the quantum determinant

$$
\begin{aligned}
\frac{\operatorname{det}_{q} \mathcal{U}_{-}(\lambda)}{\sinh (2 \lambda-2 \eta)} & =\mathcal{A}_{-}(\epsilon \lambda+\eta / 2) \mathcal{A}_{-}(\eta / 2-\epsilon \lambda)+\mathcal{B}_{-}(\epsilon \lambda+\eta / 2) \mathcal{C}_{-}(\eta / 2-\epsilon \lambda) \\
& =\mathcal{D}_{-}(\epsilon \lambda+\eta / 2) \mathcal{D}_{-}(\eta / 2-\epsilon \lambda)+\mathcal{C}_{-}(\epsilon \lambda+\eta / 2) \mathcal{B}_{-}(\eta / 2-\epsilon \lambda),
\end{aligned}
$$

where $\epsilon= \pm 1$, is a central element of the reflection algebra

$$
\left[\operatorname{det}_{q} \mathcal{U}_{-}(\lambda), \mathcal{U}_{-}(\mu)\right]=0
$$

The quantum determinant admits the following explicit expressions:

$$
\begin{aligned}
\operatorname{det}_{q} \mathcal{U}_{-}(\lambda) & =\operatorname{det}_{q} K_{-}(\lambda) \operatorname{det}_{q} M_{0}(\lambda) \operatorname{det}_{q} M_{0}(-\lambda) \\
& =\sinh (2 \lambda-2 \eta) \mathrm{A}_{-}(\lambda+\eta / 2) \mathrm{A}_{-}(-\lambda+\eta / 2),
\end{aligned}
$$

where

$$
\operatorname{det}_{q} M(\lambda)=a(\lambda+\eta / 2) d(\lambda-\eta / 2),
$$

is the bulk quantum determinant and

$$
\operatorname{det}_{q} K_{ \pm}(\lambda)=\mp \sinh (2 \lambda \pm 2 \eta) g_{ \pm}(\lambda+\eta / 2) g_{ \pm}(-\lambda+\eta / 2) .
$$

We have used here the following notations:

$$
\begin{gathered}
\mathrm{A}_{-}(\lambda)=g_{-}(\lambda) a(\lambda) d(-\lambda), \quad d(\lambda)=a(\lambda-\eta), \quad a(\lambda)=\prod_{n=1}^{N} \sinh \left(\lambda-\xi_{n}+\eta / 2\right), \\
g_{ \pm}(\lambda)=\frac{\sinh \left(\lambda+\alpha_{ \pm}-\eta / 2\right) \cosh \left(\lambda+\beta_{ \pm}-\eta / 2\right)}{\sinh \alpha_{ \pm} \cosh \beta_{ \pm}}
\end{gathered}
$$

where $\alpha_{ \pm}$and $\beta_{ \pm}$are defined in terms of the boundary parameters by:

$$
\sinh \alpha_{ \pm} \cosh \beta_{ \pm}=\frac{\sinh \zeta_{ \pm}}{2 \kappa_{ \pm}}, \quad \cosh \alpha_{ \pm} \sinh \beta_{ \pm}=\frac{\cosh \zeta_{ \pm}}{2 \kappa_{ \pm}}
$$


Proposition 2.1. The inverse monodromy matrix can be expressed in terms of the quantum determinant as follows

$$
\mathcal{U}_{-}^{-1}(\lambda+\eta / 2)=\frac{\sinh (2 \lambda-2 \eta)}{\operatorname{det}_{q} \mathcal{U}_{-}(\lambda)} \mathcal{U}_{-}(\eta / 2-\lambda)
$$

Proof. We first observe that the following identity holds

$$
K_{-}^{-1}(\lambda+\eta / 2)=\frac{\sinh (2 \lambda-2 \eta)}{\operatorname{det}_{q} K_{-}(\lambda)} K_{-}(\eta / 2-\lambda),
$$

then the identity (2.25) follows by computing the matrix products $\mathcal{U}_{-}(\eta / 2+\lambda) \mathcal{U}_{-}(\eta / 2-\lambda)$ using (2.18), (2.26) and the following identities:

$$
\hat{M}( \pm \lambda+\eta / 2)=(-1)^{\mathrm{N}} \operatorname{det}_{q} M_{0}(\mp \lambda) M^{-1}(\mp \lambda+\eta / 2) .
$$

We will also use the following properties of the generators

Proposition 2.2 (Prop. 2.1 of [36]). The generator families $\mathcal{A}_{-}(\lambda)$ and $\mathcal{D}_{-}(\lambda)$ are related by the following parity relation:

$$
\begin{aligned}
& \mathcal{A}_{-}(\lambda)=\frac{\sinh (2 \lambda-\eta)}{\sinh 2 \lambda} \mathcal{D}_{-}(-\lambda)+\frac{\sinh \eta}{\sinh 2 \lambda} \mathcal{D}_{-}(\lambda), \\
& \mathcal{D}_{-}(\lambda)=\frac{\sinh (2 \lambda-\eta)}{\sinh 2 \lambda} \mathcal{A}_{-}(-\lambda)+\frac{\sinh \eta}{\sinh 2 \lambda} \mathcal{A}_{-}(\lambda),
\end{aligned}
$$

while for the other two families the following parity relations hold:

$$
\mathcal{B}_{-}(-\lambda)=-\frac{\sinh (2 \lambda+\eta)}{\sinh (2 \lambda-\eta)} \mathcal{B}_{-}(\lambda), \quad \mathcal{C}_{-}(-\lambda)=-\frac{\sinh (2 \lambda+\eta)}{\sinh (2 \lambda-\eta)} \mathcal{C}_{-}(\lambda)
$$

It is important to mention that similar statements hold for the reflection algebra generated by $\mathcal{U}_{+}(\lambda)$. In fact, they are simply consequences of the previous proposition taking into account that $\mathcal{U}_{+}^{t_{0}}(-\lambda)$ satisfies the same reflection equation of $\mathcal{U}_{-}(\lambda)$.

For some particular choices of boundary parameters the transfer matrix is hermitian, more precisely:

Proposition 2.3 (Prop. 2.3 of [36]). The monodromy matrix $\mathcal{U}_{ \pm}(\lambda)$ satisfy the following transformation properties under Hermitian conjugation:

I) Under the condition $\eta \in i \mathbb{R}$ (massless regime), it holds:

$$
\mathcal{U}_{ \pm}(\lambda)^{\dagger}=\left[\mathcal{U}_{ \pm}\left(-\lambda^{*}\right)\right]^{t_{0}}
$$

for $\left\{i \tau_{ \pm}, i \kappa_{ \pm}, i \zeta_{ \pm}, \xi_{1}, \ldots, \xi_{\mathrm{N}}\right\} \in \mathbb{R}^{\mathrm{N}+3}$.

II) Under the condition $\eta \in \mathbb{R}$ (massive regime), it holds:

$$
\mathcal{U}_{ \pm}(\lambda)^{\dagger}=\left[\mathcal{U}_{ \pm}\left(\lambda^{*}\right)\right]^{t_{0}}
$$

for $\left\{\tau_{ \pm}, \kappa_{ \pm}, \zeta_{ \pm}, i \xi_{1}, \ldots, i \xi_{\mathrm{N}}\right\} \in \mathbb{R}^{\mathrm{N}+3}$.

Under the same conditions on the parameters of the representation it holds:

$$
\mathcal{T}(\lambda)^{\dagger}=\mathcal{T}\left(\lambda^{*}\right)
$$

i.e. $\mathcal{T}(\lambda)$ defines a one-parameter family of normal operators which are self-adjoint both for $\lambda$ real and imaginary. 
Proposition 2.4. The transfer matrix $\mathcal{T}(\lambda)$ is even in the spectral parameter $\lambda$ :

$$
\mathcal{T}(-\lambda)=\mathcal{T}(\lambda)
$$

These properties were used in [36] to construct the quantum separated variables for the boundary XXZ chain. This method can be used directly if one of the $K$ matrices is triangular. To go beyond this constraint we use the gauge transformation introduced in [7].

\section{Gauge transformations and essential properties}

\subsection{Definitions}

For arbitrary complex parameters $\alpha$ and $\beta$ we introduce the following two matrices

$$
\bar{G}(\lambda \mid \beta)=(X(\lambda \mid \beta), Y(\lambda \mid \beta)), \quad \tilde{G}(\lambda \mid \beta)=(X(\lambda \mid \beta+1), Y(\lambda \mid \beta-1))
$$

where we have defined the following columns

$$
X(\lambda \mid \beta)=\left(\begin{array}{c}
e^{-[\lambda+(\alpha+\beta) \eta]} \\
1
\end{array}\right), \quad Y(\lambda \mid \beta)=\left(\begin{array}{c}
e^{-[\lambda+(\alpha-\beta) \eta]} \\
1
\end{array}\right) .
$$

Evidently these matrices depend also on $\alpha$ but as this parameter will not vary in the following computations we omit this argument for simplicity. It is not difficult to compute the inverse matrices:

$$
\bar{G}^{-1}(\lambda \mid \beta)=\left(\begin{array}{c}
\bar{Y}(\lambda \mid \beta) \\
\bar{X}(\lambda \mid \beta)
\end{array}\right), \quad \tilde{G}^{-1}(\lambda)=\left(\begin{array}{c}
\tilde{Y}(\lambda \mid \beta-1) \\
\tilde{X}(\lambda \mid \beta+1)
\end{array}\right)
$$

in terms of the following rows

$$
\begin{gathered}
\bar{X}(\lambda \mid \beta)=\frac{e^{(\lambda+\alpha \eta)}}{2 \sinh \beta \eta}\left(1,-e^{-[\lambda+(\alpha+\beta) \eta]}\right), \\
\bar{Y}(\lambda \mid \beta)=\frac{e^{(\lambda+\alpha \eta)}}{2 \sinh \beta \eta}\left(-1, e^{-[\lambda+(\alpha-\beta) \eta]}\right) \\
\tilde{X}(\lambda \mid \beta)=e^{\eta} \frac{\sinh \beta \eta}{\sinh (\beta-1) \eta} \bar{X}(\lambda \mid \beta), \quad \tilde{Y}(\lambda)=e^{\eta} \frac{\sinh \beta \eta}{\sinh (\beta+1) \eta} \bar{Y}(\lambda \mid \beta),
\end{gathered}
$$

\subsection{Gauge transformed bulk and boundary operators}

The gauge transformation now can be applied to the local $R$-matrices. We apply it to every $R$ matrix in the auxiliary space

$$
R_{0 a}\left(\lambda-\xi_{a}-\eta / 2 \mid \beta\right)=\tilde{G}^{-1}(\lambda-\eta / 2 \mid \beta+\mathrm{N}-a) R_{0 a}\left(\lambda-\xi_{a}-\eta / 2\right) G(\lambda-\eta / 2 \mid \beta+\mathrm{N}-a+1)
$$

Now we can construct the gauge transformed bulk monodromy matrix. Taking the product of $R$ matrices as in (2.8)

$$
M(\lambda \mid \beta)=\tilde{G}^{-1}(\lambda-\eta / 2 \mid \beta) M(\lambda) \tilde{G}(\lambda-\eta / 2 \mid \beta+\mathrm{N})=\left(\begin{array}{cc}
A(\lambda \mid \beta) & B(\lambda \mid \beta) \\
C(\lambda \mid \beta) & D(\lambda \mid \beta)
\end{array}\right)
$$


where all the new monodromy matrix elements can be easily expressed in terms of the initial monodromy matrix and the row and columns defined above, for example

$$
B(\lambda \mid \beta)=\tilde{Y}(\lambda-\eta / 2 \mid \beta-1) M(\lambda) Y(\lambda-\eta / 2 \mid \beta+\mathrm{N}-1) .
$$

In a similar way we can apply the second gauge transformation to the "right to left" monodromy matrix (2.9)

$$
\hat{M}(\lambda \mid \beta)=\bar{G}^{-1}(\eta / 2-\lambda \mid \beta+\mathrm{N}) \hat{M}(\lambda) \bar{G}(\eta / 2-\lambda \mid \beta)=\left(\begin{array}{cc}
\bar{A}(\lambda \mid \beta) & \bar{B}(\lambda \mid \beta) \\
\bar{C}(\lambda \mid \beta) & \bar{D}(\lambda \mid \beta)
\end{array}\right) .
$$

We can define corresponding two-row monodromy matrix

$$
\mathrm{U}_{-}(\lambda \mid \beta)=\tilde{G}^{-1}(\lambda-\eta / 2 \mid \beta) \mathcal{U}_{-}(\lambda) \tilde{G}(\eta / 2-\lambda \mid \beta)=\left(\begin{array}{cc}
\widehat{\mathcal{A}}_{-}(\lambda \mid \beta+2) & \widehat{\mathcal{B}}_{-}(\lambda \mid \beta) \\
\widehat{\mathcal{C}}_{-}(\lambda \mid \beta+2) & \widehat{\mathcal{D}}_{-}(\lambda \mid \beta)
\end{array}\right) .
$$

Note that this definition leads to a non-trivial "dynamical" boundary bulk decomposition:

$$
\begin{aligned}
\left(\begin{array}{c}
\widehat{\mathcal{A}}_{-}(\lambda \mid \beta+2) \\
\widehat{\mathcal{C}}_{-}(\lambda \mid \beta+2)
\end{array}\right) & =M(\lambda \mid \beta) \bar{K}_{-}(\lambda \mid \beta)\left(\begin{array}{c}
\bar{A}(\lambda \mid \beta+1) \\
\bar{C}(\lambda \mid \beta+1)
\end{array}\right) \\
\left(\begin{array}{c}
\widehat{\mathcal{B}}_{-}(\lambda \mid \beta) \\
\widehat{\mathcal{D}}_{-}(\lambda \mid \beta)
\end{array}\right) & =M(\lambda \mid \beta) K_{-}(\lambda \mid \beta)\left(\begin{array}{c}
\bar{B}(\lambda \mid \beta-1) \\
\bar{D}(\lambda \mid \beta-1)
\end{array}\right),
\end{aligned}
$$

where

$$
\begin{aligned}
K_{-}(\lambda \mid \beta) & =\tilde{G}^{-1}(\lambda-\eta / 2 \mid \beta+\mathrm{N}) K_{-}(\lambda) \bar{G}(\eta / 2-\lambda \mid \beta+\mathrm{N}-1), \\
\bar{K}_{-}(\lambda \mid \beta) & =\tilde{G}^{-1}(\lambda-\eta / 2 \mid \beta+\mathrm{N}) K_{-}(\lambda) \bar{G}(\eta / 2-\lambda \mid \beta+\mathrm{N}+1) .
\end{aligned}
$$

It is more convenient to normalize the new double row monodromy matrix in the following way

$$
\mathcal{U}_{-}(\lambda \mid \beta) \equiv e^{-\lambda+\eta / 2} U_{-}(\lambda \mid \beta)=\left(\begin{array}{cc}
\mathcal{A}_{-}(\lambda \mid \beta+2) & \mathcal{B}_{-}(\lambda \mid \beta) \\
\mathcal{C}_{-}(\lambda \mid \beta+2) & \mathcal{D}_{-}(\lambda \mid \beta)
\end{array}\right)
$$

\subsection{Properties of the gauge transformed operators}

The commutation relation of the generators of the reflection algebra are given by the equation (2.2). Applying the gauge transformation one can derive the dynamical commutation relation for the transformed generators:

Lemma 3.1. The following commutations relations hold for the gauged transformed reflection algebra generators:

$$
\begin{aligned}
& \mathcal{B}_{-}\left(\lambda_{2} \mid \beta\right) \mathcal{B}_{-}\left(\lambda_{1} \mid \beta-2\right)=\mathcal{B}_{-}\left(\lambda_{1} \mid \beta\right) \mathcal{B}_{-}\left(\lambda_{2} \mid \beta-2\right) \\
& \mathcal{A}_{-}\left(\lambda_{2} \mid \beta+2\right) \mathcal{B}_{-}\left(\lambda_{1} \mid \beta\right)= \frac{\sinh \left(\lambda_{1}-\lambda_{2}+\eta\right) \sinh \left(\lambda_{2}+\lambda_{1}-\eta\right)}{\sinh \left(\lambda_{1}-\lambda_{2}\right) \sinh \left(\lambda_{1}+\lambda_{2}\right)} \mathcal{B}_{-}\left(\lambda_{1} \mid \beta\right) \mathcal{A}_{-}\left(\lambda_{2} \mid \beta\right) \\
&+\frac{\sinh \left(\lambda_{1}+\lambda_{2}-\eta\right) \sinh \left(\lambda_{1}-\lambda_{2}+(\beta-1) \eta\right) \sinh \eta}{\left.\sinh \left(\lambda_{2}-\lambda_{1}\right) \sinh \left(\lambda_{1}+\lambda_{2}\right) \sinh (\beta-1) \eta\right)} \mathcal{B}_{-}\left(\lambda_{2} \mid \beta\right) \mathcal{A}_{-}\left(\lambda_{1} \mid \beta\right) \\
&+\frac{\sinh \eta \sinh \left(\lambda_{1}+\lambda_{2}-\beta \eta\right)}{\sinh \left(\lambda_{1}+\lambda_{2}\right) \sinh (\beta-1) \eta} \mathcal{B}_{-}\left(\lambda_{2} \mid \beta\right) \mathcal{D}_{-}\left(\lambda_{1} \mid \beta\right)
\end{aligned}
$$




$$
\begin{gathered}
\mathcal{B}_{-}\left(\lambda_{1} \mid \beta\right) \mathcal{D}_{-}\left(\lambda_{2} \mid \beta\right)= \\
\quad \frac{\sinh \left(\lambda_{1}-\lambda_{2}+\eta\right) \sinh \left(\lambda_{2}+\lambda_{1}-\eta\right)}{\sinh \left(\lambda_{1}-\lambda_{2}\right) \sinh \left(\lambda_{1}+\lambda_{2}\right)} \mathcal{D}_{-}\left(\lambda_{2} \mid \beta+2\right) \mathcal{B}_{-}\left(\lambda_{1} \mid \beta\right) \\
-\frac{\sinh \left(\lambda_{2}-\lambda_{1}+(\beta+1) \eta \sinh \left(\lambda_{2}+\lambda_{1}-\eta\right)\right.}{\sinh \left(\lambda_{1}-\lambda_{2}\right) \sinh \left(\lambda_{2}+\lambda_{1}\right) \sinh (\beta+1) \eta} \mathcal{D}_{-}\left(\lambda_{1} \mid \beta+2\right) \mathcal{B}_{-}\left(\lambda_{2} \mid \beta\right) \\
-\frac{\sinh \eta \sinh \left(\lambda_{2}+\lambda_{1}+\beta \eta\right)}{\sinh \left(\lambda_{2}+\lambda_{1}\right) \sinh (\beta+1) \eta} \mathcal{A}_{-}\left(\lambda_{1} \mid \beta+2\right) \mathcal{B}_{-}\left(\lambda_{2} \mid \beta\right), \\
\mathcal{A}_{-}\left(\lambda_{1} \mid \beta+2\right) \mathcal{A}_{-}\left(\lambda_{2} \mid \beta+2\right)-\frac{\sinh \eta \sinh \left(\lambda_{1}+\lambda_{2}-(\beta) \eta\right)}{\sinh \left(\lambda_{1}+\lambda_{2}\right) \sinh (\beta-1) \eta} \mathcal{B}_{-}\left(\lambda_{1} \mid \beta\right) \mathcal{C}_{-}\left(\lambda_{2} \mid \beta+2\right)= \\
\mathcal{A}_{-}\left(\lambda_{2} \mid \beta+2\right) \mathcal{A}_{-}\left(\lambda_{1} \mid \beta+2\right)-\frac{\sinh \eta \sinh \left(\lambda_{1}+\lambda_{2}-\beta \eta\right)}{\sinh \left(\lambda_{1}+\lambda_{2}\right) \sinh (\beta-1) \eta} \mathcal{B}_{-}\left(\lambda_{2} \mid \beta\right) \mathcal{C}_{-}\left(\lambda_{1} \mid \beta+2\right) .
\end{gathered}
$$

It is not the complete list of relations that one can get from the reflection equation but it is all we need to construct the SOV representations. They can be seen as the the commutation relations of the generators of the dynamical reflection algebra [23.

It is also possible to establish symmetry properties similar to the Proposition 2.2

Proposition 3.1. The generators $\mathcal{A}_{-}(\lambda \mid \beta)$ and $\mathcal{D}_{-}(\lambda \mid \beta)$ are related by the following parity relation:

$$
\begin{aligned}
& \mathcal{A}_{-}(\lambda \mid \beta)=-\frac{\sinh \eta \sinh (2 \lambda-(\beta-1) \eta)}{\sinh 2 \lambda \sinh (\beta-2) \eta} \mathcal{D}_{-}(\lambda \mid \beta)+\frac{\sinh (2 \lambda-\eta) \sinh (\beta-1) \eta}{\sinh 2 \lambda \sinh (\beta-2) \eta} \mathcal{D}_{-}(-\lambda \mid \beta), \\
& \mathcal{D}_{-}(\lambda \mid \beta)=\frac{\sinh \eta \sinh (2 \lambda+(\beta-1) \eta)}{\sinh 2 \lambda \sinh \beta \eta} \mathcal{A}_{-}(\lambda \mid \beta)+\frac{\sinh (2 \lambda-\eta) \sinh (\beta-1) \eta}{\sinh 2 \lambda \sinh \beta \eta} \mathcal{A}_{-}(-\lambda \mid \beta),
\end{aligned}
$$

while for the other two generators the following parity relations hold:

$$
\mathcal{B}_{-}(-\lambda \mid \beta)=-\frac{\sinh (2 \lambda+\eta)}{\sinh (2 \lambda-\eta)} \mathcal{B}_{-}(\lambda \mid \beta), \quad \mathcal{C}_{-}(-\lambda \mid \beta)=-\frac{\sinh (2 \lambda+\eta)}{\sinh (2 \lambda-\eta)} \mathcal{C}_{-}(\lambda \mid \beta) .
$$

These relations can be obtained from Proposition 2.2 by direct computation.

Proposition 3.2. The inverse transformed double-row monodromy matrix can be written in terms of the quantum determinant of the reflection algebra

$$
\mathcal{U}_{-}^{-1}(\lambda+\eta / 2 \mid \beta)=\frac{\sinh (2 \lambda-2 \eta)}{\operatorname{det}_{q} \mathcal{U}_{-}(\lambda)} \mathcal{U}_{-}(\eta / 2-\lambda \mid \beta)
$$

where the following representation holds for the quantum determinant, for both $\epsilon= \pm 1$ :

$$
\begin{aligned}
\frac{\operatorname{det}_{q} \mathcal{U}_{-}(\lambda)}{\sinh (2 \lambda-2 \eta)} & =\mathcal{A}_{-}(\epsilon \lambda+\eta / 2 \mid \beta+2) \mathcal{A}_{-}(\eta / 2-\epsilon \lambda \mid \beta+2)+\mathcal{B}_{-}(\epsilon \lambda+\eta / 2 \mid \beta) \mathcal{C}_{-}(\eta / 2-\epsilon \lambda \mid \beta+2) \\
& =\mathcal{D}_{-}(\epsilon \lambda+\eta / 2 \mid \beta) \mathcal{D}_{-}(\eta / 2-\epsilon \lambda \mid \beta)+\mathcal{C}_{-}(\epsilon \lambda+\eta / 2 \mid \beta+2) \mathcal{B}_{-}(\eta / 2-\epsilon \lambda \mid \beta) .
\end{aligned}
$$

Proof. Using the definition of the gauge transformation it is easy to see that it holds:

$$
\mathcal{U}_{-}(\lambda+\eta / 2 \mid \beta)=e^{-\lambda} \tilde{G}^{-1}(\lambda \mid \beta) \mathcal{U}_{-}(\lambda+\eta / 2) \tilde{G}(-\lambda \mid \beta),
$$


then we obtain:

$$
\begin{aligned}
\mathcal{U}_{-}(\lambda+\eta / 2 \mid \beta) \mathcal{U}_{-}(\eta / 2-\lambda \mid \beta) & =\tilde{G}^{-1}(\lambda \mid \beta) \mathcal{U}_{-}(\lambda+\eta / 2) \mathcal{U}_{-}(\eta / 2-\lambda) \tilde{G}(\lambda \mid \beta) \\
& =\frac{\operatorname{det}_{q} \mathcal{U}_{-}(\lambda)}{\sinh (2 \lambda-2 \eta)},
\end{aligned}
$$

and similarly:

$$
\mathcal{U}_{-}(\eta / 2-\lambda \mid \beta) \mathcal{U}_{-}(\lambda+\eta / 2 \mid \beta)=\frac{\operatorname{det}_{q} \mathcal{U}_{-}(\lambda)}{\sinh (2 \lambda-2 \eta)} .
$$

Now the representations (3.24) and (3.25) for the quantum determinant follow directly from these expressions.

It is also easy to establish a $\beta$-parity relation for the gauged monodromy matrix

Proposition 3.3. The following identity holds:

$$
\mathcal{U}_{-}(\lambda \mid-\beta+2)=\sigma^{x} \mathcal{U}_{-}(\lambda \mid \beta) \sigma^{x}
$$

or for the matrix elements:

$$
\mathcal{B}_{-}(\lambda \mid \beta)=\mathcal{C}_{-}(\lambda \mid-\beta+2), \quad \mathcal{A}_{-}(\lambda \mid \beta)=\mathcal{D}_{-}(\lambda \mid-\beta+2) .
$$

Proof. The proof is a trivial consequence of the following simple identities:

$$
Y(\lambda \mid \beta)=X(\lambda \mid-\beta) .
$$

\subsection{Boundary transfer matrix and gauged operators}

It is possible to write the boundary transfer matrix $\mathcal{T}(\lambda)$ for the most general boundary conditions in terms of the gauged boundary operators. First we need to transform in an appropriate way the boundary matrix $K_{+}(\lambda)$. There are two possible ways to do it (we will call them left and right $K_{+}$ matrices).

We introduce two new vectors

$$
\hat{X}(\lambda \mid \beta+2)=e^{\eta} \frac{\sinh (\beta-1) \eta}{\sinh \beta \eta} X(\lambda \mid \beta+2), \quad \hat{Y}(\lambda \mid \beta-2)=e^{\eta} \frac{\sinh (\beta+1) \eta}{\sinh \beta \eta} Y(\lambda \mid \beta-2) .
$$

Then we can define the following $2 \times 2$ matrix

$$
\begin{aligned}
& K_{+}^{(L)}(\lambda \mid \beta)= \\
& \left(\begin{array}{ll}
\tilde{Y}(\eta / 2-\lambda \mid \beta-2) K_{+}(\lambda) \hat{X}(\lambda-\eta / 2 \mid \beta+2) & \tilde{Y}(\eta / 2-\lambda \mid \beta) K_{+}(\lambda) \hat{Y}(\lambda-\eta / 2 \mid \beta-2) \\
\tilde{X}(\eta / 2-\lambda \mid \beta) K_{+}(\lambda) \hat{X}(\lambda-\eta / 2 \mid \beta+2) & \tilde{X}(\eta / 2-\lambda \mid \beta+2) K_{+}(\lambda) \hat{Y}(\lambda-\eta / 2 \mid \beta-2)
\end{array}\right),
\end{aligned}
$$

the right $K_{+}$matrix is defined in a similar way

$$
K_{+}^{(R)}(\lambda \mid \beta)=\left(\begin{array}{ll}
\bar{Y}(\eta / 2-\lambda \mid \beta) K_{+}(\lambda) X(\lambda-\eta / 2 \mid \beta) & \bar{Y}(\eta / 2-\lambda \mid \beta) K_{+}(\lambda) Y(\lambda-\eta / 2 \mid \beta-2) \\
\bar{X}(\eta / 2-\lambda \mid \beta) K_{+}(\lambda) X(\lambda-\eta / 2 \mid \beta+2) & \bar{X}(\eta / 2-\lambda \mid \beta) K_{+}(\lambda) Y(\lambda-\eta / 2 \mid \beta)
\end{array}\right)
$$

The explicit expressions for these two matrices are given in the Appendix. 
Lemma 3.2. The boundary transfer matrix admits the two following representations in terms of the gauged generators:

$$
\begin{aligned}
e^{-\lambda+\eta / 2} \mathcal{T}(\lambda) & =K_{+}^{(L)}(\lambda \mid \beta-1)_{11} \mathcal{A}_{-}(\lambda \mid \beta)+K_{+}^{(L)}(\lambda \mid \beta-1)_{22} \mathcal{D}_{-}(\lambda \mid \beta) \\
& +K_{+}^{(L)}(\lambda \mid \beta-1)_{21} \mathcal{B}_{-}(\lambda \mid \beta-2)+K_{+}^{(L)}(\lambda \mid \beta-1)_{12} \mathcal{C}_{-}(\lambda \mid \beta+2),
\end{aligned}
$$

and

$$
\begin{aligned}
e^{-\lambda+\eta / 2} \mathcal{T}(\lambda) & =K_{+}^{(R)}(\lambda \mid \beta-1)_{11} \mathcal{A}_{-}(\lambda \mid \beta)+K_{+}^{(R)}(\lambda \mid \beta-1)_{22} \mathcal{D}_{-}(\lambda \mid \beta) \\
& +K_{+}^{(R)}(\lambda \mid \beta-1)_{21} \mathcal{B}_{-}(\lambda \mid \beta+2)+K_{+}^{(R)}(\lambda \mid \beta-1)_{12} \mathcal{C}_{-}(\lambda \mid \beta) .
\end{aligned}
$$

Proof. To prove the expression (3.35) we introduce a new gauge matrix

$$
\widehat{G}(\lambda \mid \beta)=(\hat{X}(\lambda \mid \beta+2), \hat{Y}(\lambda \mid \beta-2)) .
$$

It is not difficult to check that

$$
\widehat{G}^{-1}(\lambda \mid \beta)=\left(\begin{array}{c}
\tilde{Y}(\lambda \mid \beta-2) \\
\tilde{X}(\lambda \mid \beta+2)
\end{array}\right) .
$$

Now we can rewrite the right hand side of (3.35) as follows

$$
\begin{gathered}
e^{\lambda-\eta / 2}\left(\mathcal{A}_{-}(\lambda \mid \beta) K_{+}^{(L)}(\lambda \mid \beta-1)_{11}+\mathcal{B}_{-}(\lambda \mid \beta-2) K_{+}^{(L)}(\lambda \mid \beta-1)_{21}\right. \\
\left.+\mathcal{D}_{-}(\lambda \mid \beta) K_{+}^{(L)}(\lambda \mid \beta-1)_{22}+\mathcal{C}_{-}(\lambda \mid \beta+2) K_{+}^{(L)}(\lambda \mid \beta-1)_{12}\right) \\
=\tilde{Y}(\lambda-\eta / 2 \mid \beta-3) \mathcal{U}_{-}(\lambda) K_{+}(\lambda) \hat{X}(\lambda-\eta / 2 \mid \beta+1) \\
+\tilde{X}(\lambda-\eta / 2 \mid \beta+1) \mathcal{U}_{-}(\lambda) K_{+}(\lambda) \hat{Y}(\lambda-\eta / 2 \mid \beta-3) \\
=\operatorname{tr}_{0}\left\{\widehat{G}^{-1}(\lambda-\eta / 2 \mid \beta-1) \mathcal{U}_{-}(\lambda) K_{+}(\lambda) \widehat{G}(\lambda-\eta / 2 \mid \beta-1)\right\} \\
=\operatorname{tr}_{0}\left\{\mathcal{U}_{-}(\lambda) K_{+}(\lambda)\right\}=\mathcal{T}(\lambda) .
\end{gathered}
$$

The expression (3.36) can be proved in a similar way.

Proposition 3.4. The most general transfer matrix can be written in the following form

$$
\begin{aligned}
\mathcal{T}(\lambda) & =\mathrm{a}_{+}(\lambda \mid \beta-1) \mathcal{A}_{-}(\lambda \mid \beta)+\mathrm{a}_{+}(-\lambda \mid \beta-1) \mathcal{A}_{-}(-\lambda \mid \beta) \\
& +K_{+}^{(L)}(\lambda \mid \beta-1)_{21} \mathcal{B}_{-}(\lambda \mid \beta-2)+K_{+}^{(L)}(\lambda \mid \beta-1)_{12} \mathcal{C}_{-}(\lambda \mid \beta+2), \\
\mathcal{T}(\lambda) & =\mathrm{d}_{+}(\lambda \mid \beta-1) \mathcal{D}_{-}(\lambda \mid \beta)+\mathrm{d}_{+}(-\lambda \mid \beta-1) \mathcal{D}_{-}(-\lambda \mid \beta) \\
& +K_{+}^{(R)}(\lambda \mid \beta-1)_{21} \mathcal{B}_{-}(\lambda \mid \beta)+K_{+}^{(R)}(\lambda \mid \beta-1)_{12} \mathcal{C}_{-}(\lambda \mid \beta),
\end{aligned}
$$

where we have defined:

$$
\begin{aligned}
\mathrm{a}_{+}(\lambda \mid \beta) & =\frac{\sinh (2 \lambda+\eta)}{\sinh 2 \lambda \sinh (\beta-1) \eta \sinh \zeta_{+}}\left[\sinh \zeta_{+} \cosh (\lambda-\eta / 2) \sinh (\lambda+\eta / 2+\beta \eta)\right. \\
& \left.-\left(\cosh \zeta_{+} \sinh (\lambda-\eta / 2) \cosh (\lambda+\eta / 2+\beta \eta)+\kappa_{+} \sinh (2 \lambda-\eta) \sinh \left(\tau_{+}+\alpha \eta+2 \eta\right)\right)\right] \\
\mathrm{d}_{+}(\lambda \mid \beta) & =\frac{\sinh (2 \lambda+\eta)}{\sinh 2 \lambda \sinh (\beta-1) \eta \sinh \zeta_{+}}\left[\sinh \zeta_{+} \cosh (\lambda-\eta / 2) \sinh (-\lambda-\eta / 2+\beta \eta)\right. \\
& \left.-\left(\cosh \zeta_{+} \sinh (\lambda-\eta / 2) \cosh (-\lambda-\eta / 2+\beta \eta)+\kappa_{+} \sinh (2 \lambda-\eta) \sinh \left(\tau_{+}+\alpha \eta\right)\right)\right] .
\end{aligned}
$$


To prove this proposition one should use the properties of the gauged operators and the explicit form of the $K_{+}$matrices given in Appendix A.

\subsection{Reference states}

The ferromagnetic left and right states

$$
\left\langle 0\left|=\otimes_{n=1}^{\mathrm{N}}(1,0)_{n}, \quad\right| 0\right\rangle=\otimes_{n=1}^{\mathrm{N}}\left(\begin{array}{l}
1 \\
0
\end{array}\right)_{n}
$$

are no more the highest weight vectors for the spin chains with non-diagonal boundaries and it is the reason why the Bethe ansatz does not work directly for this case. However using the gauge transformation we can define new reference states which can be used in the SOV framework.

We define the following left reference state:

$$
\langle\beta| \equiv \otimes_{n=1}^{\mathrm{N}}\left(-1, e^{-\alpha \eta+(\mathrm{N}-n+\beta) \eta-\xi_{n}}\right)_{(n)}=N_{\beta}\langle 0| \prod_{n=1}^{\mathrm{N}} \bar{G}_{n}^{-1}\left(\xi_{n} \mid \beta+\mathrm{N}-n\right),
$$

where $\bar{G}_{n}^{-1}\left(\xi_{n}\right)$ is the gauge transformation acting in the local quantum space $\mathcal{H}_{n}$ and $N_{\beta}$ is a normalization factor

$$
N_{\beta}=2^{\mathrm{N}} e^{-\alpha \mathrm{N} \eta} \prod_{n=1}^{\mathrm{N}} \sinh (\mathrm{N}-n+\beta) \eta .
$$

Proposition 3.5. The state $\langle\beta|$ is a simultaneous $B(\lambda \mid \beta)$ and $\bar{B}(\lambda \mid \beta)$ left reference state:

$$
\begin{aligned}
\langle\beta| B(\lambda \mid \beta) & =\langle\beta| \bar{B}(\lambda \mid \beta)=0, \\
\langle\beta| A(\lambda \mid \beta) & =\frac{\sinh (\mathrm{N}+\beta) \eta}{\sinh \beta \eta} \prod_{n=1}^{\mathrm{N}} \sinh \left(\lambda-\xi_{n}+\eta / 2\right)\langle\beta-1|, \\
\langle\beta| D(\lambda \mid \beta) & =\prod_{n=1}^{\mathrm{N}} \sinh \left(\lambda-\xi_{n}-\eta / 2\right)\langle\beta+1|, \\
\langle\beta| \bar{A}(\lambda \mid \beta) & =\frac{\sinh \beta \eta}{\sinh (\mathrm{N}+\beta) \eta} \prod_{n=1}^{\mathrm{N}} \sinh \left(\lambda+\xi_{n}+\eta / 2\right)\langle\beta+1|, \\
\langle\beta| \bar{D}(\lambda \mid \beta) & =\prod_{n=1}^{\mathrm{N}} \sinh \left(\lambda+\xi_{n}-\eta / 2\right)\langle\beta-1| .
\end{aligned}
$$

The proposition can be checked for local $R$-matrices by direct computation.

Similarly we can define the right reference state

$$
|\beta\rangle \equiv \otimes_{n=1}^{\mathrm{N}}\left(\begin{array}{c}
e^{-\alpha \eta-(\mathrm{N}-n+\beta) \eta-\xi_{n}} \\
1
\end{array}\right)=\prod_{n=1}^{\mathrm{N}} \bar{G}_{n}\left(\xi_{n} \mid \beta+\mathrm{N}-n\right)|0\rangle,
$$

and the following proposition holds: 
Proposition 3.6. The state $|\beta+1\rangle$ is a simultaneous $C(\lambda \mid \beta)$ and $\bar{C}(\lambda \mid \beta)$ right reference state:

$$
\begin{aligned}
C(\lambda \mid \beta)|\beta+1\rangle & =\bar{C}(\lambda \mid \beta)|\beta+1\rangle=0, \\
A(\lambda \mid \beta)|\beta+1\rangle & =\prod_{n=1}^{\mathrm{N}} \sinh \left(\lambda-\xi_{n}+\eta / 2\right)|\beta+2\rangle, \\
D(\lambda \mid \beta)|\beta+1\rangle & =\frac{\sinh \eta(\mathrm{N}+\beta)}{\sinh \eta \beta} \prod_{n=1}^{\mathrm{N}} \sinh \left(\lambda-\xi_{n}-\eta / 2\right)|\beta\rangle, \\
\bar{A}(\lambda \mid \beta)|\beta+1\rangle & =\prod_{n=1}^{\mathrm{N}} \sinh \left(\lambda+\xi_{n}+\eta / 2\right)|\beta\rangle, \\
\bar{D}(\lambda \mid \beta)|\beta+1\rangle & =\frac{\sinh \eta \beta}{\sinh \eta(\mathrm{N}+\beta)} \prod_{n=1}^{\mathrm{N}} \sinh \left(\lambda+\xi_{n}-\eta / 2\right)|\beta+2\rangle .
\end{aligned}
$$

\section{SOV representations of the gauge transformed reflection algebra}

In this section we construct explicitly the SOV representation of the gauged reflection algebra. In general it is associated to the construction of the eigenstates of the operators $\mathcal{B}$ (or $\mathcal{C}$ ). However the gauge transformation and the particular structure of the reference states leads to a slightly different result. Instead of the eigenstates we construct right and left pseudo-eigenstates for these operators. More precisely, for any generic value of $\beta$ we will construct a basis in the Hilbert space $\mathcal{H}$

$$
\langle\beta, \mathbf{h}|, \quad \mathbf{h} \equiv\left(h_{1}, \ldots, h_{\mathrm{N}}\right), \quad h_{j} \in\{0,1\},
$$

formed by states that we will call left pseudo-eigenstates of $\mathcal{B}_{-}(\lambda \mid \beta)$ if they satisfy the identities

$$
\langle\beta, \mathbf{h}| \mathcal{B}_{-}(\lambda \mid \beta)=\mathbf{B}_{\mathbf{h}}(\lambda \mid \beta)\langle\beta-2, \mathbf{h}|,
$$

where for all the possible $\mathbf{h}$ the $\boldsymbol{B}_{-}(\lambda \mid \beta)$ are the pseudo-eigenvalues of $\mathcal{B}_{-}(\lambda \mid \beta)$, central elements in the algebra. Similarly we can define the basis of right pseudo-eigenstates.

The results of this section can be summarized in the following theorem.

Theorem 4.1. Let the inhomogeneities $\left\{\xi_{1}, \ldots, \xi_{\mathrm{N}}\right\} \in \mathbb{C}^{\mathrm{N}}$ satisfy the following conditions:

$$
\xi_{a} \neq \xi_{b}+r \eta \quad \forall a \neq b \in\{1, \ldots, \mathrm{N}\} \text { and } r \in\{-1,0,1\},
$$

then:

$\left.I_{b}\right)$ for any $\alpha, \beta \in \mathbb{C}$ such that for any integer $k$

$$
(\alpha-\beta) \eta \neq(\mathrm{N}-1) \eta-\tau_{-}-(-1)^{k}\left(\alpha_{-}+\beta_{-}\right)+i \pi k,
$$

the one parameter family of the gauge transformed generators of the reflection algebra $\mathcal{B}_{-}(\lambda \mid \beta)$ is left pseudo-diagonalizable and its pseudo-spectrum is simple.

$\left.I I_{b}\right)$ for any fixed $\alpha, \beta \in \mathbb{C}$ such that for any integer $k$

$$
(\alpha-\beta) \eta \neq-(\mathrm{N}+1) \eta-\tau_{-}-(-1)^{k}\left(\alpha_{-}+\beta_{-}\right)+i \pi k,
$$

the one parameter family of the gauge transformed generators of the reflection algebra $\mathcal{B}_{-}(\lambda \mid \beta)$ is right pseudo-diagonalizable and its pseudo-spectrum is simple. 
$I_{c}$ ) for any fixed $m \in \mathbb{Z}, \alpha, \beta \in \mathbb{C}$ :

$$
(\alpha+\beta) \eta \neq(\mathrm{N}+1) \eta-\tau_{-}-(-1)^{k}\left(\alpha_{-}+\beta_{-}\right)+i \pi k,
$$

the one parameter family of the gauge transformed generators of the reflection algebra $\mathcal{C}_{-}(\lambda \mid \beta)$ is left pseudo-diagonalizable and its pseudo-spectrum is simple.

$\left.I I_{c}\right)$ for any $\alpha, \beta \in \mathbb{C}$ such that for any integer $k$ :

$$
(\alpha+\beta) \eta \neq-(\mathrm{N}-1) \eta-\tau_{-}-(-1)^{k}\left(\alpha_{-}+\beta_{-}\right)+i \pi k,
$$

the one parameter family of the gauge transformed generators of the reflection algebra $\mathcal{C}_{-}(\lambda \mid \beta)$ is right pseudo-diagonalizable and its pseudo-spectrum is simple.

In all these cases we can construct a SOV representation of the gauge transformed reflection algebra.

The proof and some necessary clarifications of the statements contained in this theorem are given by the explicit constructions of the SOV representation in the next subsections. In fact, we do these constructions explicitly only for the cases $\mathrm{I}_{b}$ ) and $\mathrm{II}_{b}$ ) as for the cases $\mathrm{I}_{c}$ ) and $\mathrm{II}_{c}$ ) these constructions can be induced from the others due to the symmetries.

\section{1 $\mathcal{B}_{-}(\lambda \mid \beta)-\mathrm{SOV}$ representations of the gauge transformed reflection algebra}

\subsubsection{Left $\mathcal{B}_{-}(\lambda \mid \beta)-\mathrm{SOV}$ representations of the gauge transformed reflection algebra}

In this subsection we construct the left $\mathcal{B}_{-}(\lambda \mid \beta)$-pseudo-eigenbasis.

Theorem 4.2. Left $\mathcal{B}_{-}(\lambda \mid \beta) S O V$-basis The following states:

$$
\left\langle\beta, h_{1}, \ldots, h_{\mathrm{N}}\right|=\langle\beta| \prod_{n=1}^{\mathrm{N}}\left(\frac{\mathcal{A}_{-}\left(\eta / 2-\xi_{n} \mid \beta+2\right)}{\mathrm{A}_{-}\left(\eta / 2-\xi_{n}\right)}\right)^{h_{n}}
$$

where $\langle\beta|$ is the state defined in (3.44) and the function $\mathrm{A}_{-}$is given by (2.22). If (4.2) and (4.3) are satisfied, these states define a basis of $\mathcal{H}$ formed out of pseudo-eigenstates of $\mathcal{B}_{-}(\lambda \mid \beta)$ :

$$
\langle\beta, \mathbf{h}| \mathcal{B}_{-}(\lambda \mid \beta)=\mathbf{B}_{\mathbf{h}}(\lambda \mid \beta)\langle\beta-2, \mathbf{h}|,
$$

where $\langle\beta, \mathbf{h}|=\left\langle\beta, h_{1}, \ldots, h_{\mathrm{N}}\right|, \mathbf{h}=\left(h_{1}, \ldots, h_{\mathrm{N}}\right), h_{j} \in\{0,1\}$ and

$$
\begin{aligned}
\mathrm{B}_{\mathbf{h}}(\lambda \mid \beta)= & (-1)^{\mathrm{N}} e^{(\beta+\mathrm{N}) \eta} a_{\mathbf{h}}(\lambda) a_{\mathbf{h}}(-\lambda) \\
& \times \frac{\sinh (2 \lambda-\eta)\left(2 \kappa_{-} \sinh \left[(\mathrm{N}+\beta-\alpha-1) \eta-\tau_{-}\right]-e^{\zeta_{-}}\right)}{2 \sinh \zeta_{-} \sinh (\mathrm{N}+\beta) \eta},
\end{aligned}
$$

with

$$
a_{\mathbf{h}}(\lambda)=\prod_{n=1}^{\mathrm{N}} \sinh \left(\lambda-\xi_{n}-\left(h_{n}-\frac{1}{2}\right) \eta\right) .
$$


Proof. It is worth writing explicitly the (boundary-bulk) decomposition of the gauge transformed reflection algebra generator (3.12)

$$
\begin{aligned}
e^{\lambda-\eta / 2} \mathcal{B}_{-}(\lambda \mid \beta) & =K_{-}(\lambda \mid \beta)_{12} A(\lambda \mid \beta) \bar{D}(\lambda \mid \beta-1)+K_{-}(\lambda \mid \beta)_{11} A(\lambda \mid \beta) \bar{B}(\lambda \mid \beta-1) \\
& +K_{-}(\lambda \mid \beta)_{21} B(\lambda \mid \beta) \bar{B}(\lambda \mid \beta-1)+K_{-}(\lambda \mid \beta)_{22} B(\lambda \mid \beta) \bar{D}(\lambda \mid \beta-1) .
\end{aligned}
$$

Then, the formulae (3.46, 3.50) imply that $\langle\beta|$ is a $\mathcal{B}_{-}(\lambda \mid \beta)$-pseudo-eigenstate with non-zero eigenvalue:

$$
\langle\beta| \mathcal{B}_{-}(\lambda)=\mathrm{B}_{\mathbf{0}}(\lambda \mid \beta)\langle\beta-2|,
$$

where:

$$
\mathrm{B}_{\mathbf{0}}(\lambda \mid \beta)=(-1)^{\mathrm{N}} e^{-\lambda+\eta / 2} K_{-}(\lambda \mid \beta)_{12} a_{\mathbf{0}}(\lambda) a_{\mathbf{0}}(-\lambda),
$$

$a_{\mathbf{0}}(\lambda)$ is given by (4.10) for all $h_{j}=0$ and:

$$
e^{-\lambda+\eta / 2} K_{-}(\lambda \mid \beta)_{12}=\frac{e^{(\beta+\mathrm{N}) \eta} \sinh (2 \lambda-\eta)\left(2 \kappa_{-} \sinh \left[(\mathrm{N}+\beta-\alpha-1) \eta-\tau_{-}\right]-e^{\zeta_{-}}\right)}{2 \sinh (\mathrm{N}+\beta) \eta \sinh \zeta_{-}} .
$$

Now by using the reflection algebra commutation relations we can follow step by step the proof given in [36] to prove the validity of (4.8). Under the condition (4.2), these relations also imply that the set of states $\langle\beta, \mathbf{h}|$ forms a set of $2^{\mathrm{N}}$ independent states, i.e. a $\mathcal{B}_{-}(\lambda \mid \beta)$-pseudo-eigenbasis of $\mathcal{H}$. The action of $\mathcal{A}_{-}\left(\zeta_{b}^{\left(h_{b}\right)} \mid \beta+2\right)$ for $b \in\{1, \ldots, 2 \mathrm{~N}\}$ follows by the definition of the states $\langle\beta, \mathbf{h}|$, the reflection algebra commutation relations (3.17), the quantum determinant relations and the conditions:

$$
\langle\beta| \mathcal{A}_{-}\left(\xi_{n}-\eta / 2 \mid \beta+2\right)=0, \quad\langle\beta| \mathcal{A}_{-}\left(\eta / 2-\xi_{n} \mid \beta+2\right) \neq 0
$$

which trivially follows from the boundary-bulk decomposition (3.11)

$$
\begin{aligned}
e^{\lambda-\eta / 2} \mathcal{A}_{-}(\lambda \mid \beta+2) & =\bar{K}_{-}(\lambda \mid \beta)_{11} A(\lambda \mid \beta) \bar{A}(\lambda \mid \beta+1)+\bar{K}_{-}(\lambda \mid \beta)_{12} A(\lambda \mid \beta) \bar{C}(\lambda \mid \beta+1) \\
& +\bar{K}_{-}(\lambda \mid \beta)_{21} B(\lambda \mid \beta) \bar{A}(\lambda \mid \beta+1)+\bar{K}_{-}(\lambda \mid \beta)_{22} B(\lambda \mid \beta) \bar{C}(\lambda \mid \beta+1) .
\end{aligned}
$$

It is important to point out that the states $\langle\beta, \mathbf{h}|$ are well defined non-zero states and their definition does not depend on the order of operator $\mathcal{A}_{-}\left(\eta / 2-\xi_{b} \mid \beta+2\right)$ in their definition as it follows from the commutation relations (3.19).

Theorem 4.3. The action of the reflection algebra generators $\mathcal{A}_{-}(\lambda \mid \beta+2)$ on the generic state $\langle\beta, \mathbf{h}|$, is given by the following expression

$$
\begin{aligned}
\langle\beta, \mathbf{h}| \mathcal{A}_{-}(\lambda \mid \beta+2) & =\sum_{a=1}^{2 \mathrm{~N}} \frac{\sinh (2 \lambda-\eta) \sinh \left(\lambda+\zeta_{a}^{\left(h_{a}\right)}\right)}{\sinh \left(2 \zeta_{a}^{\left(h_{a}\right)}-\eta\right) \sinh 2 \zeta_{a}^{\left(h_{a}\right)}} \\
& \times \prod_{\substack{b=1 \\
b \neq a}}^{\mathrm{N} \text { modN }} \frac{\cosh 2 \lambda-\cosh 2 \zeta_{b}^{\left(h_{b}\right)}}{\cosh 2 \zeta_{a}^{\left(h_{a}\right)}-\cosh 2 \zeta_{b}^{\left(h_{b}\right)}} \mathrm{A}_{-}\left(\zeta_{a}^{\left(h_{a}\right)}\right)\langle\beta, \mathbf{h}| T_{a}^{-\varphi_{a}} \\
& +\operatorname{det}_{q} M(0) \cosh (\lambda-\eta / 2) \prod_{b=1}^{\mathrm{N}} \frac{\cosh 2 \lambda-\cosh 2 \zeta_{b}^{\left(h_{b}\right)}}{\cosh \eta-\cosh 2 \zeta_{b}^{\left(h_{b}\right)}}\langle\beta, \mathbf{h}| \\
& +(-1)^{\mathrm{N}+1} \operatorname{coth} \zeta_{-} \operatorname{det}_{q} M(i \pi / 2) \sinh (\lambda-\eta / 2) \prod_{b=1}^{\mathrm{N}} \frac{\cosh 2 \lambda-\cosh 2 \zeta_{b}^{\left(h_{b}\right)}}{\cosh \eta+\cosh 2 \zeta_{b}^{\left(h_{b}\right)}}\langle\beta, \mathbf{h}|,
\end{aligned}
$$


where $h_{n+\mathrm{N}} \equiv h_{n} \in\{0,1\}$, and

$$
\begin{gathered}
\zeta_{n}^{\left(h_{n}\right)}=\varphi_{n}\left[\xi_{n}+\left(h_{n}-\frac{1}{2}\right) \eta\right] \quad \forall n \in\{1, \ldots, 2 \mathrm{~N}\}, \\
\varphi_{a}=1 \quad \text { for } a \leq \mathrm{N} \quad \text { and } \quad \varphi_{a}=-1 \quad \text { for } \quad a>\mathrm{N},
\end{gathered}
$$

and:

$$
\left\langle\beta, h_{1}, \ldots, h_{a}, \ldots, h_{\mathrm{N}}\right| T_{a}^{ \pm}=\left\langle\beta, h_{1}, \ldots, h_{a} \pm 1, \ldots, h_{\mathrm{N}}\right| .
$$

Proof. Using the identities:

$$
\mathcal{U}_{-}(\eta / 2)=\operatorname{det}_{q} M(0) I_{0}, \quad \mathcal{U}_{-}(\eta / 2+i \pi / 2)=i \operatorname{coth} \zeta_{-} \operatorname{det}_{q} M(i \pi / 2) \sigma_{0}^{z},
$$

and

$$
\tilde{Y}(0 \mid \beta-1) X(0 \mid \beta+1)=1, \quad \tilde{Y}(i \pi / 2 \mid \beta-1) \sigma_{0}^{z} X(-i \pi / 2 \mid \beta+1)=-1
$$

and taking into account that $\mathcal{A}_{-}(\lambda \mid \beta)$ has the following functional dependence with respect to $\lambda$ :

$$
\mathcal{A}_{-}(\lambda \mid \beta)=\sum_{a=0}^{2 \mathrm{~N}+1} e^{(2 a-2 \mathrm{~N}+1) \lambda} \mathcal{A}_{m, a}
$$

we get the following interpolation formula for the action on $\langle\beta, \mathbf{h}|$ :

$$
\begin{aligned}
\langle\beta, \mathbf{h}| \mathcal{A}_{-}(\lambda \mid \beta+2) & =\sum_{a=1}^{2 \mathrm{~N}} \frac{\sinh (2 \lambda-\eta)}{\sinh \left(2 \zeta_{a}^{\left(h_{a}\right)}-\eta\right)} \prod_{\substack{b=1 \\
b \neq a}}^{2 \mathrm{~N}} \frac{\sinh \left(\lambda-\zeta_{b}^{\left(h_{b}\right)}\right)}{\sinh \left(\zeta_{a}^{\left(h_{a}\right)}-\zeta_{b}^{\left(h_{b}\right)}\right)} \mathrm{A}_{-}\left(\zeta_{a}^{\left(h_{a}\right)}\right)\langle\beta, \mathbf{h}| T_{a}^{-\varphi_{a}} \\
& +\operatorname{det}_{q} M(0) \cosh (\lambda-\eta / 2) \prod_{b=1}^{2 \mathrm{~N}} \frac{\sinh \left(\lambda-\zeta_{b}^{\left(h_{b}\right)}\right)}{\sinh \left(\eta / 2-\zeta_{b}^{\left(h_{b}\right)}\right)}\langle\beta, \mathbf{h}| \\
& \operatorname{coth} \zeta_{-} \operatorname{det}_{q} M(i \pi / 2) \sinh (\lambda-\eta / 2) \prod_{b=1}^{2 \mathrm{~N}} \frac{\sinh \left(\lambda-\zeta_{b}^{\left(h_{b}\right)}\right)}{\sinh \left(\eta / 2+i \pi / 2-\zeta_{b}^{\left(h_{b}\right)}\right)}\langle\beta, \mathbf{h}| .
\end{aligned}
$$

Then, it is a simple exercise to rewrite this in the form (4.17).

\subsubsection{Right $\mathcal{B}_{-}(\lambda \mid \beta)-\mathrm{SOV}$ representations of the gauge transformed reflection algebra}

Theorem 4.4. Right $\mathcal{B}_{-}(\lambda \mid \beta) S O V$-basis We define the states:

$$
\left|\beta, h_{1}, \ldots, h_{\mathrm{N}}\right\rangle=\prod_{n=1}^{\mathrm{N}}\left(\frac{\mathcal{D}_{-}\left(\xi_{n}+\eta / 2 \mid \beta\right)}{f_{n}(\beta) \mathrm{A}_{-}\left(\eta / 2-\xi_{n}\right)}\right)^{\left(1-h_{n}\right)}|-\beta+2\rangle,
$$

where:

$$
f_{n}(\beta)=\frac{\sinh \left(2 \xi_{n}+\eta\right) \sinh \beta \eta}{\sinh \left(2 \xi_{n}-\eta\right) \sinh \left(2 \xi_{n}+\beta \eta\right)},
$$


and $h_{n} \in\{0,1\}, n \in\{1, \ldots, N\}$. If (4.2) and (4.4) are satisfied, then this set of states defines a basis of $\mathcal{H}$ and they are $\mathcal{B}_{-}(\lambda \mid \beta)$ right pseudo-eigenstates:

$$
\mathcal{B}_{-}(\lambda \mid \beta)|\beta, \mathbf{h}\rangle=|\beta+2, \mathbf{h}\rangle \overline{\mathrm{B}}_{\mathbf{h}}(\lambda \mid \beta),
$$

where:

$$
\begin{aligned}
\overline{\mathrm{B}}_{\mathbf{h}}(\lambda \mid \beta)= & (-1)^{\mathrm{N}} e^{(\beta-\mathrm{N}) \eta} \prod_{n=1}^{\mathrm{N}}\left(\frac{f_{n}(\beta+2)}{f_{n}(\beta)}\right)^{1-h_{n}} a_{\mathbf{h}}(\lambda) a_{\mathbf{h}}(-\lambda) \\
& \times \frac{\sinh (2 \lambda-\eta)\left(2 \kappa_{-} \sinh \left[(\beta-(1+\mathrm{N}+\alpha)) \eta-\tau_{-}\right]-e^{\zeta_{-}}\right)}{2 \sinh \zeta_{-} \sinh \beta \eta}
\end{aligned}
$$

Proof. The proof is similar to the one for the left SOV basis. First we prove that $|-\beta+2\rangle$ is a right $\mathcal{B}_{-}(\lambda \mid \beta)$ pseudo-eigenstate. From the Proposition 3.6 and the boundary-bulk decomposition (3.11):

$$
\begin{aligned}
e^{\lambda-\eta / 2} \mathcal{C}_{-}(\lambda \mid \beta) & =\bar{K}_{-}(\lambda \mid \beta-2)_{21} D(\lambda \mid \beta-2) \bar{A}(\lambda \mid \beta-1)+\bar{K}_{-}(\lambda \mid \beta-2)_{22} D(\lambda \mid \beta-2) \bar{C}(\lambda \mid \beta-1) \\
& +\bar{K}_{-}(\lambda \mid \beta-2)_{12} C(\lambda \mid \beta-2) \bar{C}(\lambda \mid \beta-1)+\bar{K}_{-}(\lambda \mid \beta-2)_{11} C(\lambda \mid \beta-2) \bar{A}(\lambda \mid \beta-1) .
\end{aligned}
$$

It follows that the state $|\beta\rangle$ is a right $\mathcal{C}_{-}(\lambda \mid \beta)$-pseudo-eigenstate; i.e. it holds:

$$
\mathcal{C}_{-}(\lambda \mid \beta)|\beta\rangle=|\beta-2\rangle \mathbf{C}_{0}(\lambda \mid \beta)
$$

where:

$$
\mathrm{C}_{\mathbf{0}}(\lambda \mid \beta)=(-1)^{\mathrm{N}} e^{-\lambda+\eta / 2} \tilde{K}_{-}(\lambda \mid \beta)_{21} \frac{\sinh (\mathrm{N}+\beta-2) \eta}{\sinh (\beta-2) \eta} a_{\mathbf{1}}(\lambda) a_{\mathbf{1}}(-\lambda),
$$

and $a_{1}(\lambda)$ is given by (4.10) for all $h_{j}=1$ and

$$
e^{-\lambda+\eta / 2} \tilde{K}_{-}(\lambda \mid \beta)_{21}=\frac{e^{-(\beta+\mathrm{N}-2) \eta} \sinh (2 \lambda-\eta)\left(2 \kappa_{-} \sinh \left[(\mathrm{N}+\beta+\alpha-1) \eta+\tau_{-}\right]+e^{\zeta_{-}}\right)}{2 \sinh \zeta_{-} \sinh (\mathrm{N}+\beta-2) \eta} .
$$

Then from the identity (3.30), it follows that the formula (4.28) is equivalent to the following one:

$$
\mathcal{B}_{-}(\lambda \mid \beta)|-\beta+2\rangle=|-\beta\rangle \boldsymbol{C}_{0}(\lambda \mid-\beta+2) .
$$

Then by using the identities (4.30) and the commutation relations (3.18) and the formulae:

$$
\mathcal{D}_{-}\left(-\xi_{n}-\eta / 2 \mid \beta\right)|-\beta+2\rangle=0, \quad \mathcal{D}_{-}\left(\xi_{n}+\eta / 2 \mid \beta\right)|-\beta+2\rangle \neq 0,
$$

the states (4.24) are proved to be non-zero $\mathcal{B}_{-}(\lambda \mid \beta)$-pseudo-eigenstates with pseudo-eigenvalues $\overline{\mathrm{B}}_{\mathbf{h}}(\lambda \mid \beta)$ which form a basis of $\mathcal{H}$.

To define the action of the operators $\mathcal{D}_{-}(\lambda \mid \beta)$ on the generic state $|\beta, \mathbf{h}\rangle$ we will need to introduce a set of values

$$
\left.\mathrm{D}_{-}\left(\zeta_{a}^{\left(h_{a}\right)}\right)=\left[f_{a}(\beta)\right)\right]^{\varphi_{a}} \mathrm{~A}_{-}\left(-\zeta_{a}^{\left(1-h_{a}\right)}\right), \quad a=1, \ldots, 2 \mathrm{~N}
$$

It is important to underline that this set of values cannot be seen as values of some analytic function $\mathrm{D}_{-}$, however to construct the SOV representation we will need only these points. 
Theorem 4.5. The action of the reflection algebra generators $\mathcal{D}_{-}(\lambda \mid \beta)$ on the generic state $|\beta, \mathbf{h}\rangle$, can be written as follows

$$
\begin{aligned}
\mathcal{D}_{-}(\lambda \mid \beta)|\beta, \mathbf{h}\rangle= & \sum_{a=1}^{2 \mathrm{~N}} T_{a}^{-\varphi_{a}}|\beta, \mathbf{h}\rangle \frac{\sinh (2 \lambda-\eta) \sinh \left(\lambda+\zeta_{a}^{\left(h_{a}\right)}\right)}{\sinh \left(2 \zeta_{a}^{\left(h_{a}\right)}-\eta\right) \sinh 2 \zeta_{a}^{\left(h_{a}\right)}} \\
& \times \prod_{\substack{b=1 \\
b \neq a \text { modN }}}^{\mathrm{N}} \frac{\cosh 2 \lambda-\cosh 2 \zeta_{b}^{\left(h_{b}\right)}}{\cosh 2 \zeta_{a}^{\left(h_{a}\right)}-\cosh 2 \zeta_{b}^{\left(h_{b}\right)}} \mathrm{D}_{-}\left(\zeta_{a}^{\left(h_{a}\right)}\right) \\
& +|\beta, \mathbf{h}\rangle \operatorname{det}_{q} M(0) \cosh (\lambda-\eta / 2) \prod_{b=1}^{\mathrm{N}} \frac{\cosh 2 \lambda-\cosh 2 \zeta_{b}^{\left(h_{b}\right)}}{\cosh \eta-\cosh 2 \zeta_{b}^{\left(h_{b}\right)}} \\
& +(-1)^{\mathrm{N}}|\beta, \mathbf{h}\rangle \operatorname{coth} \zeta_{-} \operatorname{det}_{q} M(i \pi / 2) \sinh (\lambda-\eta / 2) \prod_{b=1}^{\mathrm{N}} \frac{\cosh 2 \lambda-\cosh 2 \zeta_{b}^{\left(h_{b}\right)}}{\cosh \eta+\cosh 2 \zeta_{b}^{\left(h_{b}\right)}}
\end{aligned}
$$

where:

$$
T_{a}^{ \pm}\left|\beta, h_{1}, \ldots, h_{a}, \ldots, h_{\mathrm{N}}\right\rangle=\left|\beta, h_{1}, \ldots, h_{a} \pm 1, \ldots, h_{\mathrm{N}}\right\rangle .
$$

Proof. The form of the action of $\mathcal{D}_{-}\left(\zeta_{a}^{\left(h_{a}\right)} \mid \beta\right)$ on $|\beta, \mathbf{h}\rangle$ is just a consequence of the definition of the states and the quantum determinant. Finally, the formula (4.33) is just a rewriting of the following interpolation formula for the action on $|\beta, \mathbf{h}\rangle$ :

$$
\begin{aligned}
\mathcal{D}_{-}(\lambda \mid \beta)|\beta, \mathbf{h}\rangle= & \sum_{a=1}^{2 \mathrm{~N}} T_{a}^{-\varphi_{a}}|\beta, \mathbf{h}\rangle \frac{\sinh (2 \lambda-\eta)}{\sinh \left(2 \zeta_{a}^{\left(h_{a}\right)}-\eta\right)} \prod_{\substack{b=1 \\
b \neq a}}^{2 \mathrm{~N}} \frac{\sinh \left(\lambda-\zeta_{b}^{\left(h_{b}\right)}\right)}{\sinh \left(\zeta_{a}^{\left(h_{a}\right)}-\zeta_{b}^{\left(h_{b}\right)}\right)} f_{a}^{\varphi_{a}}(\beta) A_{-}\left(-\zeta_{a}^{\left(1-h_{a}\right)}\right) \\
& +|\beta, \mathbf{h}\rangle \operatorname{det}_{q} M(0) \cosh (\lambda-\eta / 2) \prod_{b=1}^{2 \mathrm{~N}} \frac{\sinh \left(\lambda-\zeta_{b}^{\left(h_{b}\right)}\right)}{\sinh \left(\eta / 2-\zeta_{b}^{\left(h_{b}\right)}\right)} \\
& -|\beta, \mathbf{h}\rangle \operatorname{coth} \zeta_{-} \operatorname{det}_{q} M(i \pi / 2) \sinh (\lambda-\eta / 2) \prod_{b=1}^{2 \mathrm{~N}} \frac{\sinh \left(\lambda-\zeta_{b}^{\left(h_{b}\right)}\right)}{\sinh \left(\eta / 2+i \pi / 2-\zeta_{b}^{\left(h_{b}\right)}\right)} .
\end{aligned}
$$

\subsection{Change of basis properties}

To study the properties of the SOV basis we introduce first the standard spin basis for the 2dimensional linear space $\mathcal{H}_{n}$, the quantum space in the site $n$ of the chain,

$$
\sigma_{n}^{z}|k, n\rangle=(2 k-1)|k, n\rangle, \quad k \in\{0,1\} .
$$

Similarly, we introduce the dual $\sigma_{n}^{z}$-eigenvectors $\langle k, n|$,

$$
\langle k, n| \sigma_{n}^{z}=(2 k-1)\langle k, n|, \quad k \in\{0,1\} .
$$

The tensor products of the local basis vectors constitute an orthogonal basis in $\mathcal{H}$

$$
|\mathbf{k}\rangle=\otimes_{n=1}^{\mathrm{N}}\left|k_{n}, n\right\rangle, \quad\langle\mathbf{k}|=\otimes_{n=1}^{\mathrm{N}}\left\langle k_{n}, n\right| \quad \text { where } \quad \mathbf{k}=\left\{k_{1}, \ldots, k_{\mathrm{N}}\right\}
$$


and

$$
\left\langle\mathbf{k}^{\prime} \mid \mathbf{k}\right\rangle=\prod_{n=1}^{\mathrm{N}} \delta_{k_{n}, k_{n}^{\prime}} \quad \forall k_{n}, k_{n}^{\prime} \in\{0,1\} .
$$

We define the following $2^{\mathrm{N}} \times 2^{\mathrm{N}}$ matrices $U^{(L, \beta)}$ and $U^{(R, \beta)}$ :

$$
\langle\beta, \mathbf{h}|=\langle\mathbf{h}| U^{(L, \beta)}=\sum_{i=1}^{2^{\mathrm{N}}} U_{\varkappa(\mathbf{h}), i}^{(L, \beta)}\left\langle\varkappa^{-1}(i)\right| \quad \text { and } \quad|\beta, \mathbf{h}\rangle=U^{(R, \beta)}|\mathbf{h}\rangle=\sum_{i=1}^{2^{\mathrm{N}}} U_{i, \varkappa(\mathbf{h})}^{(R, \beta)}\left|\varkappa^{-1}(i)\right\rangle,
$$

which define the change of basis to the SOV-basis starting from the original spin basis:

$$
\langle\mathbf{h}|=\otimes_{n=1}^{\mathrm{N}}\left\langle h_{n}, n\right| \quad \text { and } \quad|\mathbf{h}\rangle=\otimes_{n=1}^{\mathrm{N}}\left|h_{n}, n\right\rangle,
$$

where $\varkappa$ is the following isomorphism between the sets $\{0,1\}^{\mathrm{N}}$ and $\left\{1, \ldots, 2^{\mathrm{N}}\right\}$ :

$$
\varkappa: \mathbf{h} \in\{0,1\}^{\mathrm{N}} \rightarrow \varkappa(\mathbf{h})=1+\sum_{a=1}^{\mathrm{N}} 2^{(a-1)} h_{a} \in\left\{1, \ldots, 2^{\mathrm{N}}\right\} .
$$

Note that the matrices $U^{(L, \beta)}$ and $U^{(R, \beta)}$ are invertible matrices for the pseudo-diagonalizability of $\mathcal{B}_{-}(\lambda \mid \beta)$ :

$$
U^{(L, \beta)} \mathcal{B}_{-}(\lambda \mid \beta)=\Delta_{\mathcal{B}_{-}}^{L}(\lambda \mid \beta) U^{(L, \beta-2)}, \quad \mathcal{B}_{-}(\lambda \mid \beta) U^{(R, \beta)}=U^{(R, \beta+2)} \Delta_{\mathcal{B}_{-}}^{R}(\lambda \mid \beta) .
$$

Here $\Delta_{\mathcal{B}_{-}}^{L / R}(\lambda \mid \beta)$ are the $2^{\mathrm{N}} \times 2^{\mathrm{N}}$ diagonal matrices with elements

$$
\left(\Delta_{\mathcal{B}_{-}}^{L}(\lambda \mid \beta)\right)_{i, j}=\delta_{i, j} \mathrm{~B}_{\varkappa^{-1}(i)}(\lambda \mid \beta), \quad\left(\Delta_{\mathcal{B}_{-}}^{R}(\lambda \mid \beta)\right)_{i, j}=\delta_{i, j} \mathrm{~B}_{\varkappa^{-1}(i)}(\lambda \mid \beta), \quad \forall i, j \in\left\{1, \ldots, 2^{\mathrm{N}}\right\} .
$$

The main result of this section is the following proposition:

Proposition 4.1. The $2^{\mathrm{N}} \times 2^{\mathrm{N}}$ matrix:

$$
M \equiv U^{(L, \beta-2)} U^{(R, \beta)}
$$

is diagonal and it is characterized by:

$$
M_{\varkappa(\mathbf{h}) \varkappa(\mathbf{k})}=\langle\beta-2, \mathbf{h} \mid \beta, \mathbf{k}\rangle=\delta_{\varkappa(\mathbf{h}) \varkappa(\mathbf{k})} Z(\beta-2) \prod_{1 \leq b<a \leq \mathrm{N}} \frac{1}{\eta_{a}^{\left(h_{a}\right)}-\eta_{b}^{\left(h_{b}\right)}},
$$

with the normalization constant

$$
Z(\beta)=\prod_{1 \leq b<a \leq \mathrm{N}}\left(\eta_{a}^{(1)}-\eta_{a}^{(1)}\right)\left\langle\beta\left|\left(\prod_{n=1}^{\mathrm{N}} \mathcal{A}_{-}\left(\eta / 2-\xi_{n} \mid \beta+2\right) / \mathrm{A}_{-}\left(\eta / 2-\xi_{n}\right)\right)\right|-\beta\right\rangle,
$$

and

$$
\eta_{a}^{\left(h_{a}\right)} \equiv \cosh 2\left[\left(\xi_{a}+\left(h_{a}-\frac{1}{2}\right) \eta\right] .\right.
$$


Proof. First we prove that the matrix $M$ is diagonal. In order to do it we compute the matrix element $\left\langle\beta, \mathbf{h}\left|\mathcal{B}_{-}(\lambda \mid \beta)\right| \beta, \mathbf{k}\right\rangle$ which lead to the following identity:

$$
\mathbf{B}_{\mathbf{h}}(\lambda \mid \beta)\langle\beta-2, \mathbf{h} \mid \beta, \mathbf{k}\rangle=\overline{\mathbf{B}}_{\mathbf{k}}(\lambda \mid \beta)\langle\beta, \mathbf{h} \mid \beta+2, \mathbf{k}\rangle,
$$

which implies:

$$
\langle\beta-2, \mathbf{h} \mid \beta, \mathbf{k}\rangle \propto \delta_{\varkappa(\mathbf{h}) \varkappa(\mathbf{k})},
$$

as from the condition $\mathbf{h} \neq \mathbf{k}$ it follows that $\exists n \in\{1, \ldots, \mathrm{N}\}$ such that $h_{n} \neq k_{n}$ and then:

$$
\mathrm{B}_{\mathbf{h}}\left(\zeta_{n}^{\left(k_{n}\right)} \mid \beta\right) \neq 0, \quad \overline{\mathrm{B}}_{\mathbf{k}}\left(\zeta_{n}^{\left(k_{n}\right)} \mid \beta\right)=0 .
$$

To compute the diagonal elements $M_{\varkappa(\mathbf{h}) \varkappa(\mathbf{h})}$, we compute the matrix elements

$$
\theta_{a}(\beta)=\left\langle\beta-2, h_{1}, \ldots, h_{a}=1, \ldots, h_{\mathrm{N}}\left|\mathcal{D}_{-}\left(\xi_{a}+\eta / 2 \mid \beta\right)\right| \beta, h_{1}, \ldots, h_{a}=0, \ldots, h_{\mathrm{N}}\right\rangle,
$$

where $a \in\{1, \ldots, \mathbf{N}\}$. Using the right action of the operator $\mathcal{D}_{-}\left(\xi_{a}+\eta / 2 \mid \beta\right)$ and the condition (4.49), we get:

$$
\begin{aligned}
\theta_{a}(\beta) & =f_{a}^{-1}(\beta) \mathrm{A}_{-}\left(\eta / 2+\xi_{a}\right) \frac{\sinh \eta}{\sinh \left(2 \xi_{a}-\eta\right)} \prod_{\substack{b=1 \\
b \neq a}}^{\mathrm{N}} \frac{\cosh 2 \zeta_{a}^{(1)}-\cosh 2 \zeta_{b}^{\left(h_{b}\right)}}{\cosh 2 \zeta_{a}^{(0)}-\cosh 2 \zeta_{b}^{\left(h_{b}\right)}} \\
& \times\left\langle\beta-2, h_{1}, \ldots, h_{a}=1, \ldots, h_{\mathrm{N}} \mid \beta, h_{1}, \ldots, h_{a}=1, \ldots, h_{\mathrm{N}}\right\rangle
\end{aligned}
$$

while using the decomposition (3.21) and the fact that:

$$
\left\langle\beta-2, h_{1}, \ldots, h_{a}=1, \ldots, h_{\mathrm{N}}\right| \mathcal{A}_{-}\left(-\left(\xi_{a}+\eta / 2\right) \mid \beta\right)=0
$$

it holds:

$$
\begin{aligned}
& \left\langle\beta-2, h_{1}, \ldots, h_{a}=1, \ldots, h_{\mathrm{N}}\right| \mathcal{D}_{-}\left(\xi_{a}+\eta / 2 \mid \beta\right) \\
& =\frac{\sinh \eta \sinh \left(2 \xi_{a}+\beta \eta\right)}{\sinh \left(2 \xi_{a}+\eta\right) \sinh (\beta) \eta}\left\langle\beta-2, h_{1}, \ldots, h_{a}=1, \ldots, h_{\mathrm{N}}\right| \mathcal{A}_{-}\left(\xi_{a}+\eta / 2 \mid \beta\right) \\
= & \frac{\sinh \eta \sinh \left(2 \xi_{a}+\beta \eta\right)}{\sinh \left(2 \xi_{a}+\eta\right) \sinh (\beta) \eta} \mathrm{A}_{-}\left(\eta / 2+\xi_{a}\right)\left\langle\beta-2, h_{1}, \ldots, h_{a}=0, \ldots, h_{\mathrm{N}}\right|,
\end{aligned}
$$

and then we get:

$$
\theta_{a}^{(m)}=\frac{\sinh \eta \sinh \left(2 \xi_{a}+(\beta) \eta\right)}{\sinh \left(2 \xi_{a}+\eta\right) \sinh (\beta) \eta} \mathrm{A}_{-}\left(\eta / 2+\xi_{a}\right)\left\langle\beta-2, h_{1}, \ldots, h_{a}=0, \ldots, h_{\mathrm{N}} \mid \beta, h_{1}, \ldots, h_{a}=0, \ldots, h_{\mathrm{N}}\right\rangle,
$$

so that it holds:

$$
\frac{\left\langle\beta-2, h_{1}, \ldots, h_{a}=1, \ldots, h_{\mathrm{N}} \mid \beta, h_{1}, \ldots, h_{a}=1, \ldots, h_{\mathrm{N}}\right\rangle}{\left\langle m-2, h_{1}, \ldots, h_{a}=0, \ldots, h_{\mathrm{N}} \mid \beta, h_{1}, \ldots, h_{a}=0, \ldots, h_{\mathrm{N}}\right\rangle}=\prod_{\substack{b=1 \\ b \neq a}}^{\mathrm{N}} \frac{\cosh 2 \zeta_{a}^{(0)}-\cosh 2 \zeta_{b}^{\left(h_{b}\right)}}{\cosh 2 \zeta_{a}^{(1)}-\cosh 2 \zeta_{b}^{\left(h_{b}\right)}}
$$

from which one can prove:

$$
\frac{\left\langle\beta-2, h_{1}, \ldots, h_{\mathrm{N}} \mid \beta, h_{1}, \ldots, h_{\mathrm{N}}\right\rangle}{\langle\beta-2,1, \ldots, 1 \mid \beta, 1, \ldots, 1\rangle}=\prod_{1 \leq b<a \leq \mathrm{N}} \frac{\eta_{a}^{(1)}-\eta_{b}^{(1)}}{\eta_{a}^{\left(h_{a}\right)}-\eta_{b}^{\left(h_{b}\right)}}
$$


This prove the proposition as it is easy to see that

$$
\langle\beta-2,1, \ldots, 1 \mid \beta, 1, \ldots, 1\rangle=Z(\beta-2) \prod_{1 \leq b<a \leq \mathrm{N}} \frac{1}{\eta_{a}^{(1)}-\eta_{b}^{(1)}},
$$

by our definition of the normalization $Z(\beta)$.

\subsection{SOV-decomposition of the identity}

The identity $\mathbb{I}$ admits the following representation in terms of left and right SOV-basis:

$$
\mathbb{I}=\sum_{i=1}^{2^{\mathrm{N}}} \mu\left|\beta, \varkappa^{-1}(i)\right\rangle\left\langle\beta-2, \varkappa^{-1}(i)\right|
$$

where the $\mu=\left(\left\langle\beta-2, \varkappa^{-1}(i) \mid \beta, \varkappa^{-1}(i)\right\rangle\right)^{-1}$ is the Sklyanin's measure 3 analogous in our 6-vertex reflection algebra representations. Now using the result of the previous section we can write it explicitly:

$$
\mathbb{I}=\frac{1}{Z(\beta-2)} \sum_{h_{1}, \ldots, h_{\mathrm{N}}=0}^{1} \prod_{1 \leq b<a \leq \mathrm{N}}\left(\eta_{a}^{\left(h_{a}\right)}-\eta_{a}^{\left(h_{a}\right)}\right)\left|\beta, h_{1}, \ldots, h_{\mathrm{N}}\right\rangle\left\langle\beta-2, h_{1}, \ldots, h_{\mathrm{N}}\right| .
$$

\section{SOV representations for $\mathcal{T}(\lambda)$-spectral problem}

In [45, 46] Sklyanin has introduced a method to construct quantum separation of variable (SOV) representations for the spectral problem of the transfer matrices associated to the representations of the Yang-Baxter algebra. For the most general representations of the reflection algebra with non-diagonal boundary matrices the quantum SOV representations are constructed here following the same approach developed in [36] but we use the gauge transformation to eliminate one of the non-diagonal entries of $K_{+}$. It means that we fix either $\alpha-\beta$ or $\alpha+\beta$. It is important to underline that the second gauge parameter remains free and can be used either to eliminate the second nondiagonal entry of $K_{+}$or the corresponding entry of $K_{-}$. However we do not need to fix this second parameter to construct the eigenvectors of the transfer matrix.

More precisely, the following theorems hold:

Theorem 5.1. Under the most general boundary conditions, and if the gauge parameters $\alpha, \beta \in \mathbb{C}$ satisfy the following condition for an integer $k$

$$
(\alpha-\beta+2) \eta=-\tau_{+}+(-1)^{k}\left(\alpha_{+}-\beta_{+}\right)+i \pi k,
$$

then $K_{+}^{(L)}(\lambda \mid \beta-1)_{12}=K_{+}^{(R)}(\lambda \mid \beta-1)_{12}=0$ and:

$\left.I_{b}\right)$ the left representation for which the one parameter family $\mathcal{B}_{-}(\lambda \mid \beta-2)$ is pseudo-diagonal defines a left $S O V$ representation for the spectral problem of the transfer matrix $\mathcal{T}(\lambda)$.

$\left.I I_{b}\right)$ the right representation for which the one parameter family $\mathcal{B}_{-}(\lambda \mid \beta)$ is pseudo-diagonal defines a right SOV representation for the spectral problem of the transfer matrix $\mathcal{T}(\lambda)$.

\footnotetext{
${ }^{3}$ Sklyanin's measure has been first introduced by Sklyanin in the quantum Toda chain [45, see also [4] and 6] for further discussions.
} 
Similarly we can formulate the same theorem for the $\mathcal{C}_{-}(\lambda \mid \beta)$ SOV representations:

Theorem 5.2. Under the most general boundary conditions, if the gauge parameters $\alpha, \beta \in \mathbb{C}$ satisfy the following condition for an integer $k$

$$
(\alpha+\beta) \eta=-\tau_{+}+(-1)^{k}\left(\alpha_{+}-\beta_{+}\right)+i \pi k,
$$

then $K_{+}^{(L)}(\lambda \mid \beta-1)_{21}=K_{+}^{(R)}(\lambda \mid \beta-1)_{21}=0$ and:

$\left.I_{c}\right)$ the left representation for which the one parameter family $\mathcal{C}_{-}(\lambda \mid \beta+2)$ is pseudo-diagonal defines a left SOV representation for the spectral problem of the transfer matrix $\mathcal{T}(\lambda)$.

$\left.I I_{c}\right)$ the right representation for which the one parameter family $\mathcal{C}_{-}(\lambda \mid \beta)$ is pseudo-diagonal defines a right SOV representation for the spectral problem of the transfer matrix $\mathcal{T}(\lambda)$.

The proof of the Theorem 5.1 and the explicit constructions of the SOV solutions of the spectral problem for the transfer matrix $\mathcal{T}(\lambda)$ will be given in the following subsections. Theorem 5.2 can be proved in a similar way.

\subsection{Transfer matrix spectrum in $\mathcal{B}_{-}(\lambda \mid \beta)-\mathrm{SOV}$-representations}

Theorem 5.3. Let $\Sigma_{\mathcal{T}}$ be the set of the eigenvalue functions of the transfer matrix $\mathcal{T}(\lambda)$, then any $\tau(\lambda) \in \Sigma_{\mathcal{T}}$ is an even function of $\lambda$ of the form:

$$
\begin{aligned}
\tau(\lambda)= & \sum_{a=1}^{\mathrm{N}} \frac{\cosh ^{2} 2 \lambda-\cosh ^{2} \eta}{\cosh ^{2} 2 \zeta_{a}^{(0)}-\cosh ^{2} \eta} \prod_{\substack{b=1 \\
b \neq a}}^{\mathrm{N}} \frac{\cosh 2 \lambda-\cosh 2 \zeta_{b}^{(0)}}{\cosh 2 \zeta_{a}^{(0)}-\cosh 2 \zeta_{b}^{(0)}} \tau\left(\zeta_{a}^{(0)}\right), \\
& +(\cosh 2 \lambda+\cosh \eta) \prod_{b=1}^{\mathrm{N}} \frac{\cosh 2 \lambda-\cosh 2 \zeta_{b}^{(0)}}{\cosh \eta-\cosh 2 \zeta_{b}^{(0)}} \operatorname{det}_{q} M(0) \\
& +(-1)^{\mathrm{N}}(\cosh 2 \lambda-\cosh \eta) \prod_{b=1}^{\mathrm{N}} \frac{\cosh 2 \lambda-\cosh 2 \zeta_{b}^{(0)}}{\cosh \eta+\cosh 2 \zeta_{b}^{(0)}} \operatorname{coth} \zeta_{-} \operatorname{coth} \zeta_{+} \operatorname{det}_{q} M(i \pi / 2) .
\end{aligned}
$$

If the condition (4.2) is satisfied, then $\mathcal{T}(\lambda)$ has simple spectrum and $\Sigma_{\mathcal{T}}$ is given by the solutions of the discrete system of equations:

$$
\tau\left( \pm \zeta_{a}^{(0)}\right) \tau\left( \pm \zeta_{a}^{(1)}\right)=\mathbf{A}\left(\zeta_{a}^{(1)}\right) \mathbf{A}\left(-\zeta_{a}^{(0)}\right), \quad \forall a \in\{1, \ldots, \mathbf{N}\},
$$

in the class of functions of the form (5.3), where the coefficient $\mathbf{A}(\lambda)$ is defined by:

$$
\mathbf{A}(\lambda) \equiv \mathbf{a}_{+}(\lambda \mid \beta-1) \mathbf{A}_{-}(\lambda),
$$

and satisfies the quantum determinant condition:

$$
\frac{\operatorname{det}_{q} K_{+}(\lambda) \operatorname{det}_{q} \mathcal{U}_{-}(\lambda)}{\sinh (2 \lambda+\eta) \sinh (2 \lambda-\eta)}=\mathbf{A}(\lambda+\eta / 2) \mathbf{A}(-\lambda+\eta / 2) .
$$

I) Under the condition (4.4), the vector:

$$
|\tau\rangle=\sum_{h_{1}, \ldots, h_{\mathrm{N}}=0}^{1} \prod_{a=1}^{\mathrm{N}} Q_{\tau}\left(\zeta_{a}^{\left(h_{a}\right)}\right) \prod_{1 \leq b<a \leq \mathrm{N}}\left(\eta_{a}^{\left(h_{a}\right)}-\eta_{b}^{\left(h_{b}\right)}\right)\left|\beta, h_{1}, \ldots, h_{\mathrm{N}}\right\rangle,
$$


defines, uniquely up to an overall normalization, the right $\mathcal{T}$-eigenstate corresponding to $\tau(\lambda) \in \Sigma_{\mathcal{T}}$. The coefficients in (5.7) are characterized by:

$$
Q_{\tau}\left(\zeta_{a}^{(1)}\right) / Q_{\tau}\left(\zeta_{a}^{(0)}\right)=\tau\left(\zeta_{a}^{(0)}\right) \mathbf{A}\left(-\zeta_{a}^{(0)}\right)
$$

II) Under the condition 4.3, the covector

$$
\langle\tau|=\sum_{h_{1}, \ldots, h_{\mathrm{N}}=0}^{1} \prod_{a=1}^{\mathrm{N}} \bar{Q}_{\tau}\left(\zeta_{a}^{\left(h_{a}\right)}\right) \prod_{1 \leq b<a \leq \mathrm{N}}\left(\eta_{a}^{\left(h_{a}\right)}-\eta_{b}^{\left(h_{b}\right)}\right)\left\langle\beta-2, h_{1}, \ldots, h_{\mathrm{N}}\right|,
$$

defines, uniquely up to an overall normalization, the left $\mathcal{T}$-eigenstate corresponding to $\tau(\lambda) \in$ $\Sigma_{\mathcal{T}}$. The coefficients in (5.9) are characterized by:

$$
\bar{Q}_{\tau_{-}}\left(\zeta_{a}^{(1)}\right) / \bar{Q}_{\tau_{-}}\left(\zeta_{a}^{(0)}\right)=\tau\left(\zeta_{a}^{(0)}\right) / \mathbf{D}\left(\zeta_{a}^{(1)}\right),
$$

where:

$$
\mathbf{D}\left(\zeta_{a}^{\left(h_{a}\right)}\right) \equiv \mathrm{d}_{+}\left(\zeta_{a}^{\left(h_{a}\right)} \mid \beta-1\right) \mathrm{D}_{-}\left(\zeta_{a}^{\left(h_{a}\right)}\right) .
$$

Proof. The transfer matrix $\mathcal{T}(\lambda)$ is an even function of $\lambda$ so the same is true for the $\tau(\lambda) \in \Sigma_{\mathcal{T}}$. Moreover, from the identities (4.21) and after some simple computation the following identities are derived:

$$
\begin{aligned}
\mathcal{T}( \pm \eta / 2) & =2 \cosh \eta \operatorname{det}_{q} M(0) \\
\mathcal{T}( \pm(\eta / 2-i \pi / 2)) & =-2 \cosh \eta \operatorname{coth} \zeta_{-} \operatorname{coth} \zeta_{+} \operatorname{det}_{q} M(i \pi / 2) .
\end{aligned}
$$

These identities together with the known functional form of $\mathcal{T}(\lambda)$ with respect to $\lambda$ imply that $\tau(\lambda) \in \Sigma_{\mathcal{T}}$ satisfy the characterization (5.3). In the $\mathcal{B}_{-}$-SOV representations the spectral problem for $\mathcal{T}(\lambda)$ is reduced to the following discrete system of $2^{\mathrm{N}}$ Baxter-like equations:

$$
\tau\left(\zeta_{n}^{\left(h_{n}\right)}\right) \Psi_{\tau}(\mathbf{h})=\mathbf{A}\left(\zeta_{n}^{\left(h_{n}\right)}\right) \Psi_{\tau}\left(\mathbf{T}_{n}^{-}(\mathbf{h})\right)+\mathbf{A}\left(-\zeta_{n}^{\left(h_{n}\right)}\right) \Psi_{\tau}\left(\mathbf{T}_{n}^{+}(\mathbf{h})\right),
$$

for any $n \in\{1, \ldots, \mathbf{N}\}$ and $\mathbf{h} \in\{0,1\}^{\mathrm{N}}$, in the coefficients (wave-functions) $\Psi_{\tau}(\mathbf{h})$ of the $\mathcal{T}$-eigenstate $|\tau\rangle$ associated to $\tau(\lambda) \in \Sigma_{\mathcal{T}}$. Here, we have used the notations:

$$
\mathbf{T}_{n}^{ \pm}(\mathbf{h})=\left(h_{1}, \ldots, h_{n} \pm 1, \ldots, h_{\mathrm{N}}\right) .
$$

This system trivially follows when we recall the identities:

$$
\mathbf{A}_{-}\left(\zeta_{n}^{(0)}\right)=\mathbf{A}_{-}\left(-\zeta_{n}^{(1)}\right)=0,
$$

and we compute the matrix elements:

$$
\left\langle\beta-2, h_{1}, \ldots, h_{n}, \ldots, h_{\mathrm{N}}\left|\mathcal{T}\left( \pm \zeta_{n}^{\left(h_{n}\right)}\right)\right| \tau\right\rangle .
$$

Indeed, from the decomposition (3.35), we have:

$$
\begin{aligned}
\tau\left( \pm \zeta_{n}^{(0)}\right) \Psi_{\tau}\left(h_{1}, \ldots, h_{n}\right. & \left.=0, \ldots, h_{\mathrm{N}}\right)= \\
& =\left\langle\beta-2, h_{1}, \ldots, h_{n}=0, \ldots, h_{\mathrm{N}}\left|\mathcal{T}\left(-\zeta_{n}^{(0)}\right)\right| \tau\right\rangle \\
& =\mathrm{a}_{+}\left(-\zeta_{n}^{(0)}\right)\left\langle\beta-2, h_{1}, \ldots, h_{n}=0, \ldots, h_{\mathrm{N}}\left|\mathcal{A}_{-}\left(-\zeta_{n}^{(0)}\right)\right| \tau\right\rangle \\
& =\mathbf{A}\left(-\zeta_{n}^{(0)}\right) \Psi_{\tau}\left(h_{1}, \ldots, h_{n}=1, \ldots, h_{\mathrm{N}}\right),
\end{aligned}
$$


and

$$
\begin{aligned}
\tau\left( \pm \zeta_{n}^{(1)}\right) \Psi_{\tau}\left(h_{1}, \ldots, h_{n}\right. & \left.=1, \ldots, h_{\mathrm{N}}\right)= \\
& =\left\langle\beta-2, h_{1}, \ldots, h_{n}=1, \ldots, h_{\mathrm{N}}\left|\mathcal{T}\left(\zeta_{n}^{(1)}\right)\right| \tau\right\rangle \\
& =\mathrm{a}_{+}\left(\zeta_{n}^{(1)}\right)\left\langle\beta-2, h_{1}, \ldots, h_{n}=1, \ldots, h_{\mathrm{N}}\left|\mathcal{A}_{-}\left(\zeta_{n}^{(1)}\right)\right| \tau\right\rangle \\
& =\mathbf{A}\left(\zeta_{n}^{(1)}\right) \Psi_{\tau}\left(h_{1}, \ldots, h_{n}=0, \ldots, h_{\mathrm{N}}\right) .
\end{aligned}
$$

Clearly the previous system of equations (5.14) is equivalent to the following system of homogeneous equations:

$$
\left(\begin{array}{cc}
\tau\left( \pm \zeta_{n}^{(0)}\right) & -\mathbf{A}\left(-\zeta_{n}^{(0)}\right) \\
-\mathbf{A}\left(\zeta_{n}^{(1)}\right) & \tau\left( \pm \zeta_{n}^{(1)}\right)
\end{array}\right)\left(\begin{array}{c}
\Psi_{\tau-}\left(h_{1}, \ldots, h_{n}=0, \ldots, h_{1}\right) \\
\Psi_{\tau-}\left(h_{1}, \ldots, h_{n}=1, \ldots, h_{1}\right)
\end{array}\right)=\left(\begin{array}{c}
0 \\
0
\end{array}\right)
$$

for any $n \in\{1, \ldots, \mathbf{N}\}$ with $h_{m \neq n} \in\{0,1\}$. The condition $\tau(\lambda) \in \Sigma_{\mathcal{T}_{-}}$implies that the determinants of the $2 \times 2$ matrices in (5.20) must be zero for any $n \in\{1, \ldots, \mathrm{N}\}$, which is equivalent to (5.4). Moreover, the rank of the matrices in (5.20) is 1 as

$$
\mathbf{A}\left(-\zeta_{n}^{(0)}\right) \neq 0 \text { and } \mathbf{A}\left(\zeta_{n}^{(1)}\right) \neq 0
$$

and then (up to an overall normalization) the solution is unique:

$$
\frac{\Psi_{\tau}\left(h_{1}, \ldots, h_{n}=1, \ldots, h_{\mathrm{N}}\right)}{\Psi_{\tau}\left(h_{1}, \ldots, h_{n}=0, \ldots, h_{\mathrm{N}}\right)}=\frac{\tau\left(\zeta_{a}^{(0)}\right)}{\mathbf{A}\left(-\zeta_{a}^{(0)}\right)},
$$

for any $n \in\{1, \ldots, \mathrm{N}\}$ with $h_{m \neq n} \in\{0,1\}$. So fixed $\tau(\lambda) \in \Sigma_{\mathcal{T}}$ there exists (up to normalization) one and only one corresponding $\mathcal{T}$-eigenstate $|\tau\rangle$ with coefficients of the factorized form given in (5.7)-(5.8); i.e. the $\mathcal{T}$-spectrum is simple.

Vice versa, if $\tau(\lambda)$ is in the set of functions (5.3) and satisfies (5.4), then the state $|\tau\rangle$ defined by (5.75.8) satisfies:

$$
\begin{aligned}
\left\langle\beta-2, h_{1}, \ldots, h_{n}, \ldots, h_{\mathrm{N}}\left|\mathcal{T}\left(\zeta_{n}^{\left(h_{n}\right)}\right)\right| \tau\right\rangle & =\left\{\begin{array}{l}
\mathbf{A}\left(-\zeta_{n}^{(0)}\right) \Psi_{\tau}\left(h_{1}, \ldots, h_{n}=1, \ldots, h_{\mathrm{N}}\right) \text { for } h_{n}=0 \\
\mathbf{A}\left(\zeta_{n}^{(1)}\right) \Psi_{\tau}\left(h_{1}, \ldots, h_{n}=0, \ldots, h_{\mathrm{N}}\right) \text { for } h_{n}=1
\end{array}\right. \\
& =\left\{\begin{array}{l}
\mathbf{A}\left(-\zeta_{n}^{(0)}\right) \frac{\tau\left(\zeta_{a}^{(0)}\right)}{\mathbf{A}\left(-\zeta_{a}^{(0)}\right)} \Psi_{\tau}\left(h_{1}, \ldots, h_{n}=0, \ldots, h_{\mathrm{N}}\right) \text { for } h_{n}=0 \\
\mathbf{A}\left(\zeta_{n}^{(1)}\right) \frac{\tau\left(\zeta_{a}^{(0)}\right)}{\mathbf{A}\left(\zeta_{a}^{(1)}\right)} \Psi_{\tau}\left(h_{1}, \ldots, h_{n}=1, \ldots, h_{\mathrm{N}}\right) \text { for } h_{n}=1
\end{array}\right. \\
& =\tau\left(\zeta_{n}^{\left(h_{n}\right)}\right) \Psi_{\tau}\left(h_{1}, \ldots, h_{n}, \ldots, h_{\mathrm{N}}\right) \forall n \in\{1, \ldots, \mathrm{N}\},
\end{aligned}
$$

this, and the following functional form with respect to $\lambda$ of the transfer matrix:

$$
\mathcal{T}(\lambda)=\sum_{b=1}^{\mathrm{N}+2} \mathcal{T}_{b}(\cosh 2 \lambda)^{b-1}
$$

implies the identity:

$$
\left\langle\beta-2, h_{1}, \ldots, h_{\mathrm{N}}|\mathcal{T}(\lambda)| \tau\right\rangle=\tau(\lambda) \Psi_{\tau}\left(h_{1}, \ldots, h_{n}, \ldots, h_{\mathrm{N}}\right) \quad \forall \lambda \in \mathbb{C},
$$


for any $\mathcal{B}_{-}(\lambda \mid \beta-2)$ pseudo-eigenstate $\left\langle\beta-2, h_{1}, \ldots, h_{\mathbf{N}}\right|$, i.e. $\tau(\lambda) \in \Sigma_{\mathcal{T}}$ and $|\tau\rangle$ is the corresponding eigenstate of the transfer matrix $\mathcal{T}$. The proof for the left $\mathcal{T}$-eigenstates is very similar and we skip it here.

Finally, it is important to point out that the quantum determinant condition (5.6) is a simple consequence of the following identity

$$
\operatorname{det}_{q} K_{+}(\lambda)=-\sinh (2 \lambda+\eta) \mathbf{a}_{+}(\lambda+\eta / 2 \mid \beta-1) \mathbf{a}_{+}(-\lambda+\eta / 2 \mid \beta-1)
$$

which can be proven by direct computations when the condition (5.1) is satisfied.

This theorem implies that each eigenvalue and eigenstate of the transfer matrix can be characterized in terms of a set of parameters $\left\{x_{1}, \ldots, x_{\mathrm{N}}\right\}$ satisfying a system of quadratic equations. This system replaces the Bethe equations in this case. More precisely:

Corollary 5.1. The set $\Sigma_{\mathcal{T}}$ of the eigenvalue functions of the transfer matrix $\mathcal{T}(\lambda)$ admits the following characterization:

$$
\Sigma_{\mathcal{T}}=\left\{\tau(\lambda): \tau(\lambda)=f(\lambda)+\sum_{a=1}^{\mathrm{N}} g_{a}(\lambda) x_{a}, \quad \forall\left\{x_{1}, \ldots, x_{\mathrm{N}}\right\} \in \Sigma_{T}\right\}
$$

where we have defined:

$$
\begin{gathered}
g_{a}(\lambda)=\frac{\cosh ^{2} 2 \lambda-\cosh ^{2} \eta}{\cosh ^{2} 2 \zeta_{a}^{(0)}-\cosh ^{2} \eta} \prod_{\substack{b=1 \\
b \neq a}}^{\mathrm{N}} \frac{\cosh 2 \lambda-\cosh 2 \zeta_{b}^{(0)}}{\cosh 2 \zeta_{a}^{(0)}-\cosh 2 \zeta_{b}^{(0)}} \quad \text { for } a \in\{1, \ldots, \mathrm{N}\}, \\
f(\lambda)=\frac{(\cosh 2 \lambda+\cosh \eta)}{2 \cosh \eta} \prod_{b=1}^{\mathrm{N}} \frac{\cosh 2 \lambda-\cosh 2 \zeta_{b}^{(0)}}{\cosh \eta-\cosh 2 \zeta_{b}^{(0)}} \tau(\eta / 2) \\
-(-1)^{\mathrm{N}} \frac{(\cosh 2 \lambda-\cosh \eta)}{2 \cosh \eta} \prod_{b=1}^{\mathrm{N}} \frac{\cosh 2 \lambda-\cosh 2 \zeta_{b}^{(0)}}{\cosh \eta+\cosh 2 \zeta_{b}^{(0)}} \tau(\eta / 2+i \pi / 2),
\end{gathered}
$$

and $\Sigma_{T}$ is the set of the solutions to the following inhomogeneous system of $\mathrm{N}$ quadratic equations:

$$
x_{n} \sum_{a=1}^{\mathrm{N}} g_{a}\left(\zeta_{n}^{(1)}\right) x_{a}+x_{n} f\left(\zeta_{n}^{(1)}\right)=q_{n}, \quad q_{n}=\frac{\operatorname{det}_{q} K_{+}\left(\xi_{n}\right) \operatorname{det}_{q} \mathcal{U}_{-}\left(\xi_{n}\right)}{\sinh \left(\eta+2 \xi_{n}\right) \sinh \left(\eta-2 \xi_{n}\right)}, \quad \forall n \in\{1, \ldots, \mathrm{N}\},
$$

in $\mathrm{N}$ parameters $\left\{x_{1}, \ldots, x_{\mathrm{N}}\right\}$.

\subsection{SOV applicability and Nepomechie's constraint}

Combining together conditions for the existence of SOV basis (4.3 4.6) and the choice of the gauge parameters necessary to construct the eigenstates of the transfer matrix (5.1- 5.2) we obtain the limits of applicability of the SOV method. It happens to be related to the constrain situation where algebraic Bethe ansatz works, more precisely the following theorem holds. 
Theorem 5.4. The SOV constructions corresponding to the cases $I_{b}$ and $I_{c}$ fails to exist if and only if the following condition on the parameters of the boundary matrices are satisfied

$$
(\mathrm{N}-1) \eta=\tau_{-}-\tau_{+}+(-1)^{k}\left(\alpha_{-}+\beta_{-}\right)-(-1)^{m}\left(\alpha_{+}-\beta_{+}\right)+i \pi(k+m),
$$

where $k$ and $m$ are arbitrary integers. Similarly, the SOV constructions corresponding to the cases $I I_{b}$ and $I I_{c}$ fails to exist if and only if the following condition on the parameters of the boundary matrices are satisfied

$$
(1-\mathrm{N}) \eta=\tau_{-}-\tau_{+}+(-1)^{k}\left(\alpha_{-}+\beta_{-}\right)-(-1)^{m}\left(\alpha_{+}-\beta_{+}\right)+i \pi(k+m) .
$$

Then our SOV schema to construct the spectrum (eigenvalues and eigenstates) of the transfer matrix $\mathcal{T}(\lambda)$ cannot be used if and only if the conditions (5.30) and (5.31) are simultaneously satisfied.

Remark In our notations for the boundary parameters the Nepomechie's constraint reads

$$
\mathrm{k} \eta=\tau_{-}-\tau_{+}+\epsilon_{-}\left(\alpha_{-}+\beta_{-}\right)+\epsilon_{+}\left(\alpha_{+}-\beta_{+}\right), \bmod 2 \pi i \text { and } \mathrm{k}=\mathrm{N}-1+2 r \text { with } r \in \mathbb{Z}
$$

so that we recover the relations (5.30) and (5.31) respectively for $r=0$ and $r=1-\mathrm{N}$. The previous theorem says that the SOV construction works also when the boundary parameters satisfy one Nepomechie's condition: if $r \neq 0$ and $r \neq 1-\mathrm{N}$ we can use both the left and right SOV construction, if $r=0$ we can use the right SOV construction and if $r=1-\mathrm{N}$ we can use the left SOV construction. The only problem in our SOV schema appears if the two Nepomechie's conditions for $r=0$ and $r=1-\mathrm{N}$ are simultaneously satisfied.

Finally, the special case when only one of these two conditions is satisfied maybe of particular interest as in this situation there are two simultaneous descriptions and it is possible to compare the construction of eigenvalues and eigenstates by the separation of variables and by the algebraic Bethe ansatz.

\section{Scalar Products}

One of the main reasons of interest in the SOV method is that it seems to provide a possibility to go beyond the spectral analysis constructing dynamic observables of the physical system. The following theorem represents the first step in the solution of this problem.

Proposition 6.1. Let $\langle\omega|$ and $|\rho\rangle$ be an arbitrary covector and vector of separate forms:

$$
\begin{aligned}
& \langle\omega|=\sum_{h_{1}, \ldots, h_{\mathrm{N}}=0}^{1} \prod_{a=1}^{\mathrm{N}} \omega_{a}\left(\zeta_{a}^{\left(h_{a}\right)}\right) \prod_{1 \leq b<a \leq \mathrm{N}}\left(\eta_{a}^{\left(h_{a}\right)}-\eta_{b}^{\left(h_{b}\right)}\right)\left\langle\beta, h_{1}, \ldots, h_{\mathrm{N}}\right|, \\
& |\rho\rangle=\sum_{h_{1}, \ldots, h_{\mathrm{N}}=0}^{1} \prod_{a=1}^{\mathrm{N}} \rho_{a}\left(\zeta_{a}^{\left(h_{a}\right)}\right) \prod_{1 \leq b<a \leq \mathrm{N}}\left(\eta_{a}^{\left(h_{a}\right)}-\eta_{b}^{\left(h_{b}\right)}\right)\left|\beta+2, h_{1}, \ldots, h_{\mathrm{N}}\right\rangle,
\end{aligned}
$$

in the $\mathcal{B}$-pseudo-eigenbasis, then the action of $\langle\omega|$ on $|\rho\rangle$ reads:

$$
\langle\omega \mid \rho\rangle=Z(\beta-2) \operatorname{det}_{\mathbb{N}}\left\|\mathcal{M}_{a, b}^{(\omega, \rho)}\right\| \quad \text { with } \mathcal{M}_{a, b}^{(\omega, \rho)}=\sum_{h=0}^{1} \omega_{a}\left(\zeta_{a}^{(h)}\right) \rho_{a}\left(\zeta_{a}^{(h)}\right)\left(\eta_{a}^{(h)}\right)^{(b-1)} .
$$

The above formula holds, in particular, if the left and right states are transfer matrix eigenstates. 
Proof. The formula (4.45) and the SOV-decomposition of the states $\langle\omega|$ and $|\rho\rangle$ implies:

$$
\langle\omega \mid \rho\rangle=Z(\beta-2) \sum_{h_{1}, \ldots, h_{\mathrm{N}}=0}^{1} V\left(\eta_{1}^{\left(h_{1}\right)}, \ldots, \eta_{\mathrm{N}}^{\left(h_{\mathrm{N}}\right)}\right) \prod_{a=1}^{\mathrm{N}} \omega_{a}\left(\zeta_{a}^{\left(h_{a}\right)}\right) \rho_{a}\left(\zeta_{a}^{\left(h_{a}\right)}\right),
$$

where

$$
V\left(x_{1}, \ldots, x_{\mathrm{N}}\right) \equiv \prod_{1 \leq b<a \leq \mathrm{N}}\left(x_{a}-x_{b}\right)
$$

is the Vandermonde determinant which due to the multilinearity of the determinant implies (6.3).

The normalization coefficient $Z(\beta-2)$ is an artifact of the gauge transformation, for any interesting quantity (form-factors, correlation functions) represented as a ratio of two scalar products this constant will disappear.

\section{Conclusion and outlook}

We have shown in this paper that the separation of variables can be applied to construct the eigenstates of the quantum spin chains with the most general boundary terms. These states are characterized by the roots of a system of $\mathrm{N}$ quadratic equations which replaces the Bethe equations in this general case. We also compute scalar products (up to an unphysical normalization constant). This representation provides a possibility to compute explicitly form factors and correlation functions.

Furthermore a very similar SOV analysis can be developed for the spectral problem of transfer matrices associated to representations of the 8-vertex reflection algebra [22] corresponding to the most general open XYZ spin chains.

After this paper was completed we became aware of the recent and interesting results reported in [8. The authors construct the $T-Q$ functional equations for the spin chains with non-diagonal boundaries 4 and thus they obtain the transfer matrix eigenvalues. An important achievement of [8] is that the equation of type (5.4) are associated to a system of Bethe equations leading to a more traditional analysis of the eigenvalue problem. It would be interesting to establish a connection between our SOV construction and this approach, in particular, with the new generalized $T-Q$ relation.

\section{Acknowledgments}

The authors would like to thank J.M. Maillet, V. Terras and K. Kozlowski for discussions. G.N. is supported by National Science Foundation grants PHY-0969739 and is grateful to the YITP Institute of Stony Brook, where he had the opportunity to develop his research programs and the privilege to have stimulating discussions with B. M. McCoy. N.K, is supported by ANR grant ANR-10-BLAN-0120-04-DIADEMS. S.F. is supported by the Burgundy region. G.N. would like to thank the Mathematical Physics Group at IMB of the Dijon University for their hospitality. G.N and N.K would like to thank the Theoretical Physics Group of the Laboratory of Physics at ENS-Lyon for hospitality. N.K. is grateful to the LPTHE laboratory (University Paris 6) for hospitality.

\footnotetext{
${ }^{4}$ See also the recent series of papers [9, 10, 11] for the application of the same method to different models.
} 


\section{A Gauge transformed boundary matrices}

We give here the explicit form of the gauge transformed boundary matrices $K_{+}^{(L)}(\lambda \mid \beta)$ and $K_{+}^{(R)}(\lambda \mid \beta)$

$$
\begin{aligned}
& K_{+}^{(L)}(\lambda \mid \beta)_{11}=\frac{1}{\sinh \beta \eta \sinh \zeta_{+}}\left[\sinh \zeta_{+} \cosh (\lambda+\eta / 2) \sinh (\lambda-\eta / 2+\beta \eta)\right. \\
&\left.-\left(\cosh \zeta_{+} \sinh (\lambda+\eta / 2) \cosh (\lambda-\eta / 2+\beta \eta)+\kappa_{+} \sinh (2 \lambda+\eta) \sinh \left(\tau_{+}+(\alpha+2) \eta\right)\right)\right] \\
& K_{+}^{(L)}(\lambda \mid \beta)_{12}=\frac{e^{(\beta+1) \eta} \sinh (2 \lambda+\eta)\left[\kappa_{+} \sinh \left((\beta-1-\alpha) \eta-\tau_{+}\right)-e^{-\zeta_{+} / 2}\right]}{\sinh \beta \eta \sinh \zeta_{+}} \\
& K_{+}^{(L)}(\lambda \mid \beta)_{21}=\frac{e^{-(\beta-1) \eta} \sinh (2 \lambda+\eta)\left[\kappa_{+} \sinh \left((\beta+\alpha+1) \eta+\tau_{+}\right)+e^{-\zeta_{+}} / 2\right]}{\sinh \beta \eta \sinh \zeta_{+}} \\
& K_{+}^{(L)}(\lambda \mid \beta)_{22}=\frac{1}{\sinh (\beta+1) \eta \sinh \zeta_{+}}\left[\sinh \zeta_{+} \cosh (\lambda+\eta / 2) \sinh (-\lambda+\eta / 2+\beta \eta)\right. \\
&\left.-\left(\cosh \zeta_{+} \sinh (\lambda+\eta / 2) \cosh (-\lambda+\eta / 2+\beta \eta)+\kappa_{+} \sinh (2 \lambda+\eta) \sinh \left((\alpha+2) \eta+\tau_{+}\right)\right)\right]
\end{aligned}
$$

and

$$
\begin{aligned}
& K_{+}^{(R)}(\lambda \mid \beta)_{11}=\frac{e^{\zeta_{+}} \sinh (\beta-1) \eta-e^{-\zeta_{+}} \sinh (2 \lambda+\beta \eta)-2 \kappa_{+} \sinh (2 \lambda+\eta) \sinh \left(\tau_{+}+\alpha \eta\right)}{2 \sinh \beta \eta \sinh \zeta_{+}} \\
& K_{+}^{(R)}(\lambda \mid \beta)_{12}=e^{-2 \eta} K_{+}^{(L)}(\lambda \mid \beta)_{12}, \quad K_{+}^{(R)}(\lambda \mid \beta)_{21}=e^{-2 \eta} K_{+}^{(L)}(\lambda \mid \beta)_{21} \\
& K_{+}^{(R)}(\lambda \mid \beta)_{22}=\frac{e^{-\zeta_{+}} \sinh (2 \lambda-\beta \eta)+e^{\zeta_{+}} \sinh (\beta+1) \eta+2 \kappa_{+} \sinh (2 \lambda+\eta) \sinh \left(\tau_{+}+\alpha \eta\right)}{2 \sinh \beta \eta \sinh \zeta_{+}} .
\end{aligned}
$$

\section{References}

[1] P. Baseilhac. The $q$-deformed analogue of the Onsager algebra: Beyond the Bethe ansatz approach Nucl. Phys. B 754 (2006) 309.

[2] P. Baseilhac, K. Koizumi. A deformed analogue of Onsager's symmetry in the XXZ open spin chain J. Stat. Mech. (2005) P10005.

[3] R. Baxter. Partition function of the eight-vertex lattice model. Ann. Phys., 70:193-228, 1972.

[4] R. J. Baxter. One-dimensional anisotropic Heisenberg chain. Ann. Phys., 70:323-37, 1972.

[5] H. Bethe. Zür Theorie der Metalle I. Eigenwerte und Eigenfunktionen Atomkete. Zeitschrift für Physik, 71:205-226, 1931.

[6] A. G. Bytsko and J. Teschner. Quantization of models with non-compact quantum group symmetry: modular XXZ magnet and lattice sinh-Gordon model. Journal of Physics A: Mathematical and General, 39(41):12927, 2006.

[7] J. Cao, H.-Q. Lin, K.-J. Shi, and Y. Wang. Exact solution of $X X Z$ spin chain with unparallel boundary fields. Nuclear Phys. B, 663(3):487-519, 2003. 
[8] J. Cao, W. Yang, K. Shi, and Y. Wang. Off-diagonal Bethe ansatz solutions of the anisotropic spin-1/2 chains with arbitrary boundary fields. arXiv preprint arXiv:1307.2023

[9] J. Cao, W. Yang, K. Shi, and Y. Wang. Off-diagonal Bethe ansatz and exact solution a topological spin ring. arXiv preprint arXiv:1305.7328, 2013.

[10] J. Cao, W. Yang, K. Shi, and Y. Wang. Off-diagonal bethe ansatz solution of the XXX spin-chain with arbitrary boundary conditions. arXiv preprint $\operatorname{arXiv:1306.1742,} 2013$.

[11] J. Cao, W. Yang, K. Shi, and Y. Wang. Spin-1/2 XYZ model revisit: general solutions via off-diagonal Bethe ansatz. arXiv preprint arXiv:1307.0280, 2013.

[12] I. V. Cherednik. Factorizing particles on a half-line and root systems. Theor. Math. Phys., 61:977-983, 1984.

[13] N. Crampé, E. Ragoucy, D. Simon. Eigenvectors of open XXZ and ASEP models for a class of non-diagonal boundary conditions J. Stat. Mech. P11038 (2010).

[14] N. Crampé, E. Ragoucy Generalized coordinate Bethe ansatz for non-diagonal boundaries Nucl. Phys. B 858 (2012) 502.

[15] J. de Gier and F. H. L. Essler. Bethe ansatz solution of the asymmetric exclusion process with open boundaries. Phys. Rev. Lett., 95(24):240601, 4, 2005.

[16] J. de Gier and F. H. L. Essler. Exact spectral gaps of the asymmetric exclusion process with open boundaries. Journal of Statistical Mechanics: Theory and Experiment, 2006(12):P12011, 2006.

[17] W. Galleas. Functional relations from the Yang?Baxter algebra: Eigenvalues of the XXZ model with non-diagonal twisted and open boundary conditions Nucl. Phys. B 790 (2008) 524.

[18] J. de Gier and P. Pyatov. Bethe ansatz for the Temperley?Lieb loop model with open boundaries J. Stat. Mech. P03002 (2004).

[19] N. Grosjean, J. M. Maillet, and G. Niccoli. On the form factors of local operators in the lattice sinegordon model. Journal of Statistical Mechanics: Theory and Experiment, P10006, 2012.

[20] N. Grosjean, G. Niccoli, The $\tau_{2}$-model and the chiral Potts model revisited: completeness of Bethe equations from Sklyanin's SOV method. J. Stat. Mech. P11005 (2012).

[21] L. D. Faddeev, E. K. Sklyanin, and L. A. Takhtajan. Quantum inverse problem method I. Theor. Math. Phys., 40:688-706, 1979.

[22] S. Faldella, G. Niccoli. SOV approach for integrable quantum models associated to the most general representations on spin-1/2 chains of the 8 -vertex reflection algebra. to appear.

[23] G. Filali and N. Kitanine. Spin chains with non-diagonal boundaries and trigonometric SOS model with reflecting end. SIGMA Symmetry Integrability Geom. Methods Appl., 7:Paper 012, 22, 2011. 
[24] H. Frahm, A. Seel, T. Wirth. Separation of variables in the open XXX chain. Nucl. Phys. B $802(2008) 351$.

[25] H. Frahm, J. H. Grelik, A. Seel, T. Wirth. Functional Bethe ansatz methods for the open XXX chain. J. Phys. A 44 (2011) 015001.

[26] N. Kitanine, K. Kozlowski, J. Maillet, G. Niccoli, N. Slavnov, and V. Terras. On correlation functions of the open $X X Z$ chain I. J. Stat. Mech.: Theory Exp., pages P10009, 37 pp. (electronic), 2007.

[27] N. Kitanine, K. Kozlowski, J. Maillet, G. Niccoli, N. Slavnov, and V. Terras. On correlation functions of the open $X X Z$ chain II. J. Stat. Mech.: Theory Exp., page P07010, 2008. arXiv:0803.3305.

[28] N. Kitanine, K. Kozlowski, J. Maillet, N. Slavnov, and V. Terras. A form factor approach to the asymptotic behavior of correlation functions in critical models. Journal of Statistical Mechanics: Theory and Experiment, 2011(12):P12010, 2011.

[29] N. Kitanine, K. K. Kozlowski, J. M. Maillet, N. A. Slavnov, and V. Terras. Form factor approach to dynamical correlation functions in critical models. J. Stat. Mech. Theory Exp., pages P09001, 33, 2012.

[30] N. Kitanine, J. M. Maillet, and V. Terras. Form factors of the $X X Z$ Heisenberg spin-1/2 finite chain. Nucl. Phys. B, 554 [FS]:647-678, 1999.

[31] N. Kitanine, J. M. Maillet, and V. Terras. Correlation functions of the $X X Z$ heisenberg spin-1/2 chain in a magnetic field. Nucl. Phys. B, 567 [FS]:554-582, 2000.

[32] J. M. Maillet and V. Terras. On the quantum inverse scattering problem. Nucl. Phys. B, 575:627, 2000. Preprint LPENSL-TH-19/99, hep-th/9911030.

[33] R. Murgan and R. I. Nepomechie. Bethe ansatz derived from the functional relations of the open XXZ chain for new special cases. J. Stat. Mech., (2005) P08002.

[34] R. I. Nepomechie. Solving the open XXZ spin chain with nondiagonal boundary terms at roots of unity. Nuclear Physics B, 622(3):615 - 632, 2002.

[35] R. I. Nepomechie. Bethe ansatz solution of the open $X X Z$ chain with nondiagonal boundary terms. J. Phys. A, 37(2):433-440, 2004. Special issue on recent advances in the theory of quantum integrable systems.

[36] G. Niccoli. Non-diagonal open spin-1/2 XXZ quantum chains by separation of variables: complete spectrum and matrix elements of some quasi-local operators. J. Stat. Mech.: Theory and Exp., 2012(10):P10025, 2012.

[37] G. Niccoli. Antiperiodic spin-1/2 XXZ quantum chains by separation of variables: Complete spectrum and form factors. Nucl.Phys. B, 870: $397-420,2013$.

[38] G. Niccoli. Form factors and complete spectrum of XXX antiperiodic higher spin chains by quantum separation of variables. J. Math. Phys. 54, 053516 (2013). 
[39] G. Niccoli. Antiperiodic dynamical 6-vertex model I: Complete spectrum by SOV, matrix elements of the identity on separate states and connections to the periodic 8-vertex model. J. Phys. A: Math. Theor. 46 075003, 2013.

[40] G. Niccoli and J. Teschner. The sine-Gordon model revisited: I. J. Stat. Mech.: Theory Exp., P09014 (2010).

[41] A. Nichols, V. Rittenberg and J. de Gier. One-boundary Temperley?Lieb algebras in the XXZ and loop models. Stat. Mech. P03003 (2005).

[42] T. Prosen. Open XXZ spin chain: Nonequilibrium steady state and a strict bound on ballistic transport. Phys. Rev. Lett., 106:217206, May 2011.

[43] J. Sirker, R. G. Pereira, and I. Affleck. Diffusion and ballistic transport in one-dimensional quantum systems. Phys. Rev. Lett., 103:216602, Nov 2009.

[44] E. Sklyanin. Boundary conditions for integrable quantum systems. J. Phys. A : Math. Gen., 21:2375-2389, 1988.

[45] E. K. Sklyanin. The quantum Toda chain. In Nonlinear equations in classical and quantum field theory (Meudon/Paris, 1983/1984), volume 226 of Lecture Notes in Phys., pages 196233. Springer, Berlin, 1985.

[46] E. K. Sklyanin. Quantum inverse scattering method. selected topics. In M.-L. Ge, editor, Quantum group and Quantum Integrable Systems, pages 63-97. Nankai Lectures in Mathematical Physics, World Scientific, 1992.

[47] N. A. Slavnov. Calculation of scalar products of wave functions and form factors in the framework of the algebraic Bethe ansatz. Theor. Math. Phys., 79:502-508, 1989.

[48] F. A. Smirnov. Structure of matrix elements in the quantum Toda chain. J. Phys. A : Math. Gen., 31(44):8953-8971, 1998.

[49] L. A. Takhtajan and L. D. Faddeev. The quantum method of the inverse problem and the heisenberg XYZ model. Russ. Math. Surveys, 34(5):11-68, 1979.

[50] W.-L. Yang and Y.-Z. Zhang. On the second reference state and complete eigenstates of the open $X X Z$ chain. J. High Energy Phys., pages 044, 11 pp. (electronic), 2007. 